\title{
Lipid and Nucleic Acid Chemistries: Combining the Best of Both Worlds to Construct Advanced Biomaterials
}

\author{
Julie Baillet $t^{+}$Valérie Desvergnes ${ }^{t}$, Aladin Hamoud, Laurent Latxague, and Philippe \\ Barthélémy*
}

Julie Baillet, Dr. Valérie Desvergnes, Aladin Hamoud, Dr. Laurent Latxague, Pr. Philippe Barthélémy

ARNA Laboratory, Inserm, U1212, CNRS 5320,

Université de Bordeaux, F-33000, Bordeaux, France.

E-mail: philippe.barthelemy@inserm.fr

$\$$ Julie Baillet and Valérie Desvergnes contributed equally to this work.

Keywords: nucleosides, lipids, amphiphiles, supramolecular assemblies, hydrogels

\begin{abstract}
Hybrid synthetic amphiphilic biomolecules are emerging as promising supramolecular materials for biomedical and technological applications. Herein, we highlight recent progress in the field of nucleic acid-based lipids with an emphasis on their molecular design, synthesis, supramolecular properties, physico-chemical behaviors and applications in the field of health science and technology. In the first section, we focus on the design and the study of nucleolipids and then discuss the glyconucleolipids family. In the last section, we describe recent contributions on responsive materials involving nucleolipids and their use as smart drug delivery systems. The supramolecular materials generated by nucleic acid-based lipids open new challenges for biomedical applications, including in the fields of medicinal chemistry, biosensors, biomaterials for tissue engineering, drug delivery and the decontamination of nanoparticles.
\end{abstract}

\section{Introduction}

In the last twenty years, research on materials for biomedical applications has been mainly focused on the fabrication of materials that confer properties of interest (biocompatibility, biodegradability, stiffness, and sustainability, among others) to a device or a system. To meet 
the material specifications, a fundamental principle has been to modulate the material properties by assembling polymers together. We are currently witnessing a paradigm shift with novel materials derived from non-polymeric small molecules that can adapt to the environment and actively perform tasks depending on the local conditions and/or stimuli. In the field of biomedicine, the next generation of materials will be designed to actively perform complex functions or tasks in biological environments. Nature provides many examples of such materials, which are composed of supramolecular assemblies involving biomolecules and which include nucleic acids, peptides, oligosaccharides, lipids, etc. In this regard, bioinspired supramolecular materials designed using basic supramolecular principles, including hydrogen bonding, metal chelation, hydrophobic effects, $\pi-\pi$ interactions, and/or van der Waals interactions, are of major interest for application in the field of health science and technology.

Interestingly, synthetic biomolecules can mimic many properties of their biological analogs. Hence, novel bioinspired chemical structures are currently under investigation in order to address a broad range of demands in different areas of biomedicine, including molecular science and nanoscience, regenerative medicine, drug delivery and bioimaging. In this regard, materials designed via the synthetic combination of biological units, such as lipids, nucleic acids and/or sugars, represent a simple approach to create new functionalities and new biological mechanical properties. Here, recent advances in the synthesis and applications of hybrid nucleolipids and glyconucleolipids will be described, with a particular attention toward their molecular, supramolecular and biological properties. Some potential uses of these hybrid biomolecules within the disciplines of nanotechnologies and supramolecular biomaterials will also be highlighted.

In this contribution, we will review the recent progress in the synthesis and study of hybrid nucleolipids. We will mainly focus on nucleolipids (NLs, nucleoside and nucleotide-lipids) 
and glyconucleolipids (GNLs and bolaamphiphiles), as we believe these families present promising potential in the field of health science and technology. We will further highlight recent contributions involving responsive NLs and impressive applications of these materials in soft material engineering and drug delivery. Finally, some anticipated challenges related to the development of the next generation of nucleic acid-based lipids will be discussed.

\section{Nucleolipids}

Generally, NLs are hybrid molecules combining a nucleobase, nucleoside, and nucleotide with a lipophilic part (Figure 1). ${ }^{[1-4]}$ Hybrid lipid-nucleoside or nucleotide compounds can be natural, occurring in eucaryotic and procaryotic cells. During the last several years, synthetic chemists have devoted efforts combining the potential biological functions and activities of nucleosides and nucleotides with the self-assembling properties of lipids. Originally, NLs were designed and synthesized to investigate the impact of molecular structural modifications on the physico-chemical and subsequently aggregation properties. A wide panel of novel amphiphilic NLs has been reported. They can be classified according various criteria, including charge, substitution position (2' and 3', 5', onto the base), structural composition (nucleoside or nucleotide) or shape (bola form for example). Synthetic methods providing both nucleoside lipid and nucleotide lipid conjugates and their applications reported since 2010 will be first explored herein. Approaches involving lipid oligonucleotides (LON) will not be discussed in this contribution; reviews on these compounds have been published previously. ${ }^{[5-7]}$ The following reported compounds have been selected on the basis of their structural originality, for their interest as new materials or for further potential applications.

\subsection{Nucleoside lipids}

\subsubsection{Nucleoside lipids synthesized via esters, amides, ether, amine or sulfur functions}


Since 2010, only a few approaches synthesizing nucleoside lipids have been reported. Most of them involve very simple linker functions such as esters, ethers or amide links at various positions on the sugar ring $\left(2^{\prime}, 3^{\prime}, 5^{\prime}\right)$ or anchored on the nucleobase.

In 2011, polyacylated thymidine or uridine-based NLs were synthesized and studied for potential applications as nanovectors in drug delivery. ${ }^{[8]}$ To induce self-assembly in aqueous solutions, NLs were built from a thymidine or uridine central scaffold bearing a pyridine arm inserted at the $N-3$ position of the base, a hydrophilic oligo(ethylene glycol) linked at the 5'$\mathrm{OH}$ ribose group expected to optimize the hydrophilic/lipophilic balance, and one or two (in the case of uridine) lipophilic oleyl chains attached to the ribose secondary hydroxyl group(s) (Figure 2). Classical coupling reaction conditions were used to introduce first the oleyl residue at the 2'-OH position and secondly the oligo(ethylene glycol) chain at the 5'-OH position. For that purpose, triethylene glycol (TEG) and hexaethylene glycol (HEG) were used as starting materials for the acid derivatives (Figure 2A). ToThy 2, HoThy $\mathbf{3}$ and DoHu 4 compounds were obtained in good overall yields using a four-step sequence (41$48 \%$ ) and dissolved in pure water (or phosphate buffer) to allow the formation of large aggregates, which were analyzed by dynamic light scattering (DLS). Under pseudophysiological conditions, the presence of multilayer vesicles was highlighted. The radius of these vesicles was smaller in the buffer than when measured in pure water, where the presence of salts enhanced the formation of packed structures of alkyl chains (Figure 2B). Bioactivity and in vitro cytotoxicity were investigated, revealing the absence of significant toxicity on both normal and tumor cells for HoThy 3 and DoHu 4, making them both very interesting candidate carriers to incorporate metal complex-based drugs using the pyridine arm as a ligand (Figure 2C).

To go further, and following the same approach on a thymidine central scaffold, Montesarchio and coll. used the pyridine methyl arm as a ruthenium ligand to provide an octaedric complex 
with the $\mathrm{Ru}(\mathrm{III})$ cation (Figure 3). The AziRu bioactive complex was then inserted into a nucleoside-lipid scaffold (Figure 3A frame). The synthesis of an original cholesterol-based thymidine-NL ruthenium complex named ToThyCholRu 5, stabilized by lipid aggregates for antineoplastic therapy, was reported (Figure 3A). ${ }^{[9]}$ Indeed, ruthenium derivatives have emerged as promising alternatives to Pt-based cisplatin chemotherapeutic drugs. ${ }^{[10]}$ To increase its stability in aqueous medium, this original ToThyCholRu ruthenium complex $\mathbf{5}$ was incorporated into the phospholipid membrane of a liposome bilayer formed by the naturally occurring lipid 1-palmitoyl-2-oleyl-sn-glycero-3-phosphocholine (POPC), leading to a lesser degradation of Ru aggregates. As shown in Figure 3B, the cholesterol residue was inserted inside the bilayer, whereas the metal $\mathrm{Ru}$ head was lodged between phospholipid hydrophilic heads. The effects of ToThyCholRu/POPC liposomes on the cellular viability of two human cancer cell lines (WiDr epithelial colorectal adenocarcinoma cells and MCF-7 breast adenocarcinoma cells) were explored. With lower $\mathrm{IC}_{50}$ values (the half maximal inhibitory concentration), ToThyCholRu/POPC exhibited a better inhibiting activity against human cancer cells than the reference Ru-complex, AziRU (Figure 3C). This exciting result demonstrates that nucleoside lipid structures or scaffolds can improve the anticancer activity of drugs.

A series of ruthenium-based complex nanocarriers were subsequently designed for cancer therapy. ToThyRu 6 and HoThyRu 7, represented below, are based on a thymidine structure, and DoHuRu 8 on a uridine structure (Figure 4). ${ }^{[1]}$ The multifunctional nucleoside scaffold was decorated with one or two oleic acid chains (2'- and 3'-positions) and with an oligo(ethylene oxide) at the 5'-position allowing for amphiphilic properties at the resulting edifice (Figure 4A). Ruthenium complex derivatives were found to exhibit self-aggregation behavior, forming liposomes among other things. DLS studies suggested that ternary systems exhibited the presence of a single translational diffusive species (Figure 4B). The average 
$\sim 60 \mathrm{~nm} R_{H}$ value was observed to be in the same range as the one obtained for pure POPCbased liposomes. This suggested that there was no micelle formation and that $\mathrm{Ru}$ complexes were inserted into the liposome bilayer without leading to destabilization. Interestingly, their stable formulations in POPC, as innovative vectorization systems, exhibited highly promising in vitro antiproliferative activities on different cancer cell lines (MCF-7 human breast adenocarcinoma cells, WiDr human colorectal adenocarcinoma cells and Tumor C6 rat glioma cells).

To take full advantage of the nucleobase recognition ability, Montesarchio and coll. developed the synthesis of a second generation of NL-based Ru complexes where the pyridine ligand was not anchored at the $N-3$ position but at the 3 '-position of the sugar moiety (Figure 5). ${ }^{[12]}$ The key intermediate here was the 3'-azido-3'-deoxy-1- $\beta$-Dxylofuranosyluracil 9, ${ }^{[13]}$ which was substituted following the same global modular strategy, as previously described, to provide the HoUrRu $\mathbf{1 0}$ complex in a 7-step sequence and with a $35 \%$ overall yield.

This compound was shown to co-aggregate with either POPC (up to 15 mol\%) or DOTAP ( $N$ [1-(2,3-Dioleoyloxy)propyl]- $N, N, N$-trimethylammonium chloride) (up to $50 \mathrm{~mol} \%$ ) to provide stable formulations in physiological medium. POPC/HoUrRu and DOTAP/HoUrRu aggregates have been characterized by DLS, small-angle neutron scattering (SANS) and electron paramagnetic resonance (EPR) analyses. It was highlighted that HoUrRu displayed an enhanced ability to co-aggregate with various lipids. Moreover, the in vitro inhibition properties of aggregates against human cancer cells MCF-7 and WiDr proved to be much more effective than the reference AziRu complex. It also exhibited the best activity against WiDr cells compared to previously cited Ru complexes. Such nucleoside lipid-Ru complexes are undoubtedly very original and promising in vivo drug carriers. ${ }^{[14]}$ 
In 2010, Drummond and coll. reported the synthesis of non-ionic thymidine-based NLs: 3'oleylthymidine 11, 3'-phytanoylthymidine 12, and the study of their physical properties (Figure 6A). ${ }^{[15]}$ In particular, their phase behavior in aqueous media was explored using microscopy techniques and small-angle X-ray scattering (SAXS). As expected, the hydroxyl group at the 5'-position in 3'-oleylthymidine 11 and in 3'-phytanoylthymidine $\mathbf{1 2}$ seemed to be essential for the polar head hydrophobicity in water, which led to a lyotropic phase at room temperature. In contrast, the disubstituted analog of compound 11, bearing two oleyl moieties at the 3'- and 5'-positions, was non-water swelling under the same conditions. Transmission electron microscopy (TEM) of spherulites from 3'-oleylthymidine 11 showed fibrils (Figure 6B). The authors concluded that regarding to their size and surface area/volume ratio, such fibril organizations should be useful to provide carrier vehicles for encapsulation and the controlled release of agents. The ability of the synthetic derivatives to bind single-stranded DNA oligomers in a monolayer film form was explored using a Langmuir trough (Figure 6C). Thymidine NLs were evaluated for their specificity toward adenosine complementary 10-base oligomers. The behavior of both 3'-substituted compounds was found to be similar. The molecular surface at monolayer collapse in the presence of DNA oligomers (polyadenosine and polythymidine) was shown to increase, indicating interaction between monolayers of both 3'-monosubstituted NLs and DNA oligomers at the air-water interface. This interaction emphasis is particularly promising for the use of these readily accessible simple NLs as transfection agents.

Liebscher and coll. reported in 2010 a straightforward synthesis of 2'-substituted uridine 13ac using a $\mathrm{Cu}$-catalyzed azide-alkyne cycloaddition $(\mathrm{CuAAC})$ to introduce various groups of biological interest such as lipids, fluorophores, lysine, biotin or glycoside (Figure 7). ${ }^{[16]}$ Spectroscopic studies were used to investigate the anchorage of cholesterol-derivatives into POPC membranes. 
The distribution of the different sugar, uridine and triazole parts of the NL in the membrane was investigated using two-dimensional ${ }^{1} \mathrm{H}$ magic angle spinning (MAS) NOESY NMR spectroscopy. Cross-relaxation rate values revealed that they were localized within the lipid/water interface region of the membrane. NMR spectroscopy investigations probed the anchorage of 2'-triazole substituted NLs in lipid membranes without affecting the molecular order in bilayers.

Very recently, Srivatsan and coll. reported the synthesis of very simple thymidine-based NLs as innovative materials with surface tunability and metal ion responsiveness (Figure 8A). ${ }^{[17]}$ Two types of fatty acid substituted NLs were synthesized and studied. 3',5'-O-disubstituted compounds were found to exhibit organogelation properties in various pure organic solvents (Figure 8C). Conversely, a mixture of water and organic solvent was required to induce the formation of stable gels from 3'-monosubstituted NLs (Figure 8B). An exception could be noticed in the case of unsaturated oleyl chain, which did not gel regardless of the solvent conditions used. The morphology of these various xerogels was studied using field emission scanning electron microscopy (FESEM) showing the formation of long sheets for 3'monosubstituted NLs (Figure 8B). Interestingly, 3',5'-O-disubstituted compounds formed long ribbons indicating that different gelation mechanisms were involved (Figure 8C left). Thy crystal structure analysis, power X-ray diffraction (PXRD), and NMR studies indicated that for 3'-O-palmitoyl NLs, hydrophobic effects between lipidic tails and polar interactions between nucleobases combined with hydrogen bonding with water were responsible for the gelation properties. It was demonstrated that the nature of the solvent mixture used for gelation, influencing the possible orientation of fatty tails, was crucial in the surface property tuning, which was efficiently switched from hydrophobic to hydrophilic (Figure 8B right). Introduction of small amounts of $\mathrm{Hg}^{2+}$ cations was shown to disrupt 3',5'-O-distearoyl thymidine gelation, which was clearly visible by the degradation of twisted long ribbons on 
scanning electron microscopy (SEM) images (Figure 8C right). NMR analyses indicated that the cation should have displaced the $\mathrm{H}$ bonding involved in the supramolecular assembling to form a thymidine-Hg-thymidine pair. This work represents a very nice example of how the supramolecular assemblies can be tuned by taking advantage of nucleobase-metal ion interactions. Indeed, besides nucleobases-nucleobases specific or canonic interactions, it is well known that certain nucleobases interact specifically with ions. For example, thymidine provides thymidine-Hg(II)-thymidine pairs, whereas cytosine interacts specifically with silver to give cytosine- $\mathrm{Ag}(\mathrm{I})$-cytosine pairs. Hence, thanks to the presence of specific nucleobases in the NL structures, one can control the supramolecular systems by simply introducing a specific metal ion.

Regarding nucleoside lipids connected via ether linkage, several structures have been investigated. Rosemeyer's team has extensive experience in the synthesis and biological evaluation of NLs. A part of their recent work included the use of 5-fluorouridine $\mathbf{1 4}$ as a starting material for lipidic prodrug derivative synthesis (Figure 9A). Indeed, a large number of lipophilic prodrugs of 5-fluorouracil and its nucleoside derivatives have been found to exhibit antitumor activity. In this context, Rosemeyer and coll. also reported access to a series of cyclic 15a-g and acyclic $O-2$ ', 3'-ketal derivatives 16a-c using various cyclic and linear ketones and triethylorthoformate as reagents under classical acidic conditions. ${ }^{[18]}$

The lipophilicity of each synthetic derivative was investigated by the calculation of the $\log P$, Parachor and Amphiphilic ratios. It was demonstrated that the calculated $\log P$ values increased proportionately with the number of $\mathrm{CH}_{2}$ groups. Their stability in acidic conditions was also described. Toxicity using an MTT assay was also evaluated, highlighting that largering ketals $(12 \mathrm{C}, \mathrm{n}=11) \mathbf{1 5 f}$ were highly toxic.

A similar synthetic approach was previously used to investigate the formation of selfassemblies resulting from interactions of complementary ketal-based adenosine and uridine 
nucleoside lipids. ${ }^{[19]}$ Interestingly, mixing complementary ketal-based nucleosides provided new stable supramolecular systems due to complementary adenosine-uridine recognition. ${ }^{[20]}$ These results validated the idea that self-assemblies could be driven via molecular recognition events between nucleoside lipids, which is a fundamental concept in supramolecular chemistry. These results are likely to be of interest for programmable supramolecular engineering.

\subsubsection{Approach toward nucleobase-substituted nucleolipids}

Mitsunobu approach to afford anchorage at the N-3 position of 5-fluorouridine

In 2013, Rosemeyer and coll. reported the synthesis of nucleoterpenes in which 5fluorouridine was substituted at the $N-3$ position by either phytol or nerol using a Mitsunobu reaction (Figure 9B). ${ }^{[21]}$ 5-fluorouridine 14 was first protected at the $2^{\prime}, 3^{\prime}-\mathrm{OH}$ positions as previously described (Figure 9A) and then at the 5'-OH position before being submitted to the Mitsunobu reaction with either phytol or nerol $\left(\mathrm{PPh}_{3}, \mathrm{DEAD}\right.$ diethylazodicarboxylate in THF). The resulting NLs were then transformed into corresponding 5'-phosphoramidite compounds 17a-b to provide oligonucleotides bearing terminal phytol-alkylated 5fluorouridine tags for further studies.

\section{Nucleoterpenes of thymidine and 2'-deoxyinosine}

To date, only one natural meronucleoterpene has been reported (avinosol), isolated from the sponge Dysidea. Its structure is based on a $\mathrm{N}-1$ alkylated 2'-deoxyinosine derivative bearing a sesquiterpene residue and was found to exhibit anti-angiogenic and antimetastatic properties. Seeking inspiration from such a structure, Rosemeyer and coll. reported in 2013 the first synthesis of biomimetic nucleoterpenes of thymidine and 2'-deoxyinosine (Figure 10). ${ }^{[22]}$ Indeed, thymidine and 2'-deoxyinosine were easily $N$-alkylated using $\mathrm{K}_{2} \mathrm{CO}_{3}$ in $\mathrm{DMF}$ and, 
alternatively, geranylbromide and farnesylbromide to provide the $N$-alkylated derivatives 18ab, 19a-b, which were subsequently converted into corresponding 3'-O-phosphoramidites. These phosphoramidites were used to prepare lipophilized oligonucleotides dodecamers, which were then inserted into artificial membranes (1-palmitoyl-2-oleyl-glycero-3-phosphoethanolamine POPE and POPC).

Following a similar synthetic pathway, Rosemeyer and coll. also reported the synthesis of $N-3$ alkylated 2',3'-O-ethyl levulinate derivatives and corresponding acids from 5fluorouridine 20a-b (Figure 11A). ${ }^{[23]}$ Farnesylated NLs were then coupled to various chitosans (different sources and molecular weight) to provide covalent immobilization in homogeneous solution and on chitosan foils. ATTO-488 was covalently linked to the polymer as a fluorescent dye, and the degradation of the 5-fluorouridine bound polymer under the action of chitosanane enzyme was investigated. Figure 11B displays the absorbance of the acceptor-cell content of the Franz diffusion cell. After a total degradation time of 240 hours, the end of the reaction was indicated by the same measured absorbance at $268 \mathrm{~nm}$ in both compartments of the diffusion cell $(268 \mathrm{~nm}$ being the characteristic wavelength for uridine absorption).

The purpose of this experiment was to study the potential of such polymers as biomedical materials for the sustained release of a cancerostatic drug (5-fluorouridine) and in the topical treatment of dermatological affections by taking advantage of chitosan biocompatibility with human skin.

The cytotoxic and cytostatic activities of 5-fluorouridine derivatives $\mathbf{2 0 a}-\mathbf{b}$ and 21 toward three carninoma cell lines, colon 5 (HT-29), hepatocellular (HepG2) and renal (RENCA), were also investigated by Rosemeyer and coll. ${ }^{[24]}$ Indeed, as 5-fluorouridine efficiency is well known for chemotherapy against various cancers, it is very quickly metabolized in the liver. Then, the use of structurally modified derivatives, particularly lipophilic derivatives, should 
be interesting tools to investigate new modes of drug administration. Concerning HT-29 human cells, after treatment for 48 hours, $20 b(\mathrm{R}=\mathrm{Et})$ and $\mathbf{2 1}$ were found to exhibit significant inhibitions of survival at $80 \mu \mathrm{M}$ compared to 5-fluorouridine. At the same concentration and concerning HePG2 cells, 20b and 21 were respectively shown to be 3.7and 2.5-fold more efficient than the 5-fluorouridine reference. 20b also exhibited significantly better activity against the murine RENCA cell line than the reference 5-fluorouridine. Cytotoxic effects of both compounds on the viability of PMA (phorbol myristate acetate)differentiated human THP-1 macrophages were investigated as an in vitro model to make a distinction between anticancer and side effects. If $\mathbf{2 1}$ proved to significantly increase the cytotoxicity, 20b was found to increase the viability compared to 5-fluorouridine. This study is rather encouraging for the use of lipophilic nucleosides as antitumor agents.

Farnesylated NL derivatives (20b $\mathrm{R}=\mathrm{Et}$ in particular) were labeled with the coumarine fluorophore ATTO-425® to follow their potential incorporation and intracellular localization. ${ }^{[25]}$ These compounds were also tested as cytostatic/cytotoxic agents using HT-29 human colon carcinoma cells, in comparison with 5-fluorouracil and 5-fluorouridine.

In 2011, Srivatsan and coll. reported the synthesis of fluorescent pyrimidine ribonucleoside analog 22 bearing benzofuran onto the 5-position of uracil (Figure 12A top). ${ }^{[26]}$ For all the compounds described, the synthetic pathway involved a Stille pallado-catalyzed crosscoupling approach between commercially available 5-iodouridine and a stannane derivative. Time-resolved fluorescence spectroscopic analyses were performed to investigate the effect of solvent polarity on the excited-state decay kinetics of 22 (Figure 12B). In water, the benzofurane derivative exhibited the highest lifetime of $2.55 \mathrm{~ns}$. This ability to respond to polarity changes and its spectral domain of emission made it a good candidate as a fluorescent probe to study nucleic acid structures. For this purpose, the 5-(benzofuran-2yl)pyrimidine analog 22 was subsequently converted into the corresponding triphosphate derivative $\mathbf{2 3}$ 
(Figure 12A bottom), which was efficiently incorporated into oligoribonucleotides by T7 RNA polymerase, affording fluorescent oligoribonucleotide objects. Spectroscopic studies of these oligonucleotides established that the chromophore on the ribonucleoside monomer interacted with neighbor bases involving subtle changes in its environment and then a photophysical response to these changes. It was highlighted that after incorporation into an oligomer, the emissive ribonucleoside preferably indicated a DNA abasic site with an increase of fluorescence intensity.

At the same time, and using the same synthetic pathway, Srivatsan and coll. reported the synthesis, characterization and use of a uridine nucleoside thiophene analog and its desoxyuridine analog. ${ }^{[27]}$

The same approach was used very recently to provide NL hybrids incorporating emissive nucleosides following very classical coupling reaction conditions with a variety of lipidic chains before the Stille reaction step (Figure 13A). ${ }^{[28]}$ Fluorescent derivatives were shown to exhibit a good gelation ability in organic solvents. The influence of self-assembly onto the photophysical behavior of these NLs was also investigated. Four of these NLs provided organogels easily, and it is interesting to note that contrary to most of the previously described low molecular weight gelators, these compounds exhibited enhanced emission upon selforganization (Figure 13B). NLs 25b and 26b containing myristoyl chains self-assembled into nanotubes, whereas $\mathbf{2 5 c}$ and $\mathbf{2 6 c}$, containing palmitoyl acyl chains, provided twisted ribbons and fiber organization.

It was highlighted that the gelation of these hybrid materials was made reversible by external stimuli, such as temperature, ultrasound or chemicals, promising interesting potential applications in the field of optical materials or probes.

\subsection{Nucleotide lipids}


Among non-viral vectors for therapeutic acid nucleic delivery, supramolecular assemblies of amphiphile molecules have emerged as interesting alternative tools. In particular, nucleotide lipids, taking full advantage of selective interactions between nucleic bases of both partners (NLs and nucleic acids), are prime non-toxic candidates. Two main nucleotide lipid families have been investigated, including phosphate groups attached to either the 3'- or 5'-positions of the ribose moiety (Figure 1).

\subsubsection{5'-phosphate nucleotide lipids}

Regarding 5'-phosphate derivatives, Berti and coll. reported in 2012 the formation of NLbased anionic liposomes from POP-Ade:POPC (1-palmitoyl-2-oleoyl-phosphatidyladenosine and 1-palmitoyl-2-oleoyl-phosphatidylcholine respectively) and their association with singlestranded and double-stranded nucleic acids. ${ }^{[29]}$ Interestingly, they demonstrated that specific interactions occurring between nucleotide lipids and single-strand oligonucleotides allowed the formation of hybrid complexes. However, no association was observed in the case of lipid samples of POPG (1-palmitoyl-2-oleoyl-sn-phosphatidyl-glycerol). These types of cooperative interactions, driven by molecular recognition, were previously observed both in solution $^{[30]}$ and in low water content regimes. ${ }^{[31]}$ More recently, Berti and coll. reported the ability of NL bilayers to selectively bind single-stranded nucleic acids. ${ }^{[32]}$ In another study, the binding between nucleotide lipid/dendrimer surfaces and oligonucleotides was reported. $^{[33,34]}$ This system featured cationic PAMAM dendrimers (Figure 14A), which formed films with negatively charged nucleotide lipids. As shown in Figure 14, functionalized surfaces selectively bound nucleic acids depending on the type of nucleotide head group. In that example, nucleotide lipids (DLPNs, Figure 14B) were attached to preadsorbed PAMAM monolayers on silica and produced layers with similar structures and 
composition featuring favorable nucleobases orientation. In a second step, after the addition of nucleic acid, selective interactions with nucleic acids through base stacking and specific base pairing could occur (Figure 14C). According to Nylander and coll., this type of functionalized surface can find applications in the field of bioanalytic devices or as new drug delivery systems.

As mentioned in the previous section, lipid nucleoside prodrugs feature important anticancer activities. Similarly, nucleotide analogs have been recently investigated as antitumor conjugates. To address drawbacks, such as low bioavailability, poor pharmacokinetic properties and the toxic effects of nucleotide drugs, lipid prodrugs have been synthesized by Tsybulskaya and coll. ${ }^{[35]}$ Lipid conjugates of the anticancer nucleoside clofarabine [2-chloro9-(2-deoxy-2-fluoro- $\beta$-D-arabinofuranosyl)adenine $] \quad$ with $\quad 1,2-\quad$ and $1,3-$ diacylglycerophosphates have been prepared via the phosphoramidite method. Interestingly, nucleotide lipid conjugates exhibited cytostatic activity against HL-60, A-549, MCF-7, and HeLa tumor cell lines. More recently, Berti and coll. investigated the self-assembly behavior of two diastereoisomers of clofarabine dioxy-ether nucleotide lipids. ${ }^{[36]}$ The authors demonstrated that these derivatives showed a diastereo selective self-assembly effect, which would play a role in the different diastereoisomer bioavailabilities.

At the fundamental level, nucleotide lipids have been studied as part of protocell models. Vesicles coming from the supramolecular self-assembly of NLs could be considered as prebiotic component or cell precursors. Recently, Herdewijn and coll. reported a series of $\alpha / \beta$ hydroxy fatty acids and $\alpha$-amino fatty acids, covalently bound to nucleoside-5monophosphates. ${ }^{[37]}$ They demonstrated the potential of these compounds to spontaneously self-assemble into spherical aggregates. The ability of these compounds to act as activated or reactive monomers for the "spontaneous" synthesis of oligonucleotides in abiotic conditions was investigated. An NMR study revealed that phosphoesters were cleaved between sugar and 
phosphate. However, no oligomerization of nucleotides was observed, indicating that these $\alpha-$ hydroxy or $\alpha$-amino nucleotide lipids could not induce the formation of phosphodiester linkages.

\subsubsection{3'-phosphate nucleotide lipids}

The modulation of the nucleobase structure, in particular the spatial orientation of nucleobases relative to the lipid chain, can have an impact on the aggregation properties of nucleotide lipids. Barthélémy and coll. have been working on a series of 3'-nucleotide lipid analogs, including monoalkyl phosphate ${ }^{[38,39]}$ and 1,2-diacyl-sn-glycerol derivatives ${ }^{[40]}$, as lipid moieties with a phosphate group attached to the 3'-secondary hydroxyl. Additionally, nucleotide-3'-monophosphate bolaamphiphile structures have been previously reported by Shimizu and coll. ${ }^{[41]}$

Regarding colloidal properties, 1,2-diacyl-sn-glycerol derivatives (Figure 15A) have been investigated in different conditions, including at the air-water interface of Langmuir films, ${ }^{[42]}$ vesicular systems, ${ }^{[43]}$ or gels ${ }^{[44]}$. A study of Langmuir isotherms performed in 2012 revealed the formation of a quasi-hexagonal packing of bilayer domains at a low compression rate. This unexpected behavior for lipids at the air-water interface was not previously reported for regular phospholipids (Figure 15B). This observation indicated that the 3'-phosphate nucleotide polar head impacted on supramolecular assemblies. Dispersions of 27b-c in HEPES buffer led to the stabilization of unilamellar vesicles, whereas multilamellar vesicles were observed in the same conditions for amino acid nucleotide lipid conjugates $\mathbf{2 7 b}$ '-c' (Figure 15C). The different behaviors observed could be explained by additional interactions between bilayers in the case of amino acid conjugates. Recently, hydrogels resulting from the self-assembly of $\mathrm{DiC}_{16^{-}} 3^{\text {'-dT }}$ 27a in the presence of particular counter ions $\left(\mathrm{Li}^{+}, \mathrm{Na}^{+}\right.$and $\left.\mathrm{K}^{+}\right)$ were reported by Barthélémy's group (Figure 15D). ${ }^{[44]}$ These biocompatible soft scaffolds, 
exhibiting elastic, injectability (thixotropic), and thermal reversibility properties, were investigated for drug delivery applications.

Self-assembly properties of NLs interacting via specific interactions with oligonucleotides have long been studied in different systems and combinations. ${ }^{[45]}$ To improve these interactions, locked nucleic acid (LNA)-based nucleotide lipids featuring 3'-monophosphate thymidine or adenosine head groups covalently attached to 1,2-dipalmitoyl-sn-glycerol (DiC 16 -3'-LNA-T and $\mathrm{DiC}_{16}$-3'-LNA-A, respectively) were synthesized. ${ }^{[46]}$ As expected, LNA adenosine lipid ( $\mathrm{DiC}_{16}$-3'-LNA-A) exhibited a high binding affinity for polyuridine with the formation of a stable complex $(\mathrm{Kd} \approx 43 \mathrm{nM})$. In theory, such a strategy involving specific interactions can be used for novel therapeutic strategies (for example, nucleotide lipid as RNA-binders).

1,2-diacyl-sn-glycerol nucleotide lipids have been used for several biomedical applications (Figure 16). For example, in the field of drug delivery, these molecules allow the efficient encapsulation and delivery of cisplatin. It was demonstrated that nucleoside polar heads guided the aggregate self-assembly into highly loaded and stable nanoparticles such as those displayed in Figure 16B. Interestingly, nucleotide-based nanoparticles were efficient vehicles for the delivery of cisplatin into different sensitive and resistant cancer cell lines. ${ }^{[47]}$ In another contribution, nucleotide lipids were used to stabilize solid lipid nanoparticles (SLNs) loaded with iron oxide particles and therapeutic agents (Figure 16C). ${ }^{[48]}$ It was demonstrated that nucleotide lipid-based SLNs featured high relaxivity properties, indicating the suitability of SLNs for image-guided therapy. As seen above, 27a self-assembled into ribbon-like supramolecular structures stabilizing low molecular weight hydrogels (Figure 16D). This system displayed thixotropy, allowing for its in vivo injectability. A thixotropic gel can be defined as a gel that is thick or viscous under static conditions and can become fluid (less viscous) over time when shaken, sheared or stressed. Importantly, this type of gel possesses 
self-healing properties and recovers its initial gel state, with initial rheological properties (elastic G' and viscous G' moduli) intact when the strain is removed. Thus, this bioinspired supramolecular material was injected in vivo to form an in situ hydrogel, allowing a sustained release of small drugs and proteins for more than a week. ${ }^{[44]}$ Such injectable non-polymeric materials represent a promising option to address several key challenges for in vivo sustained release of drugs or biologics such as a biocompatible 3D matrix for stem cell growth.

Thus, the potential of NLs (under different supramolecular formats as nanoparticles, vesicles, surfaces, complexes or hydrogels) in biomedical applications, including drug delivery, tissue engineering, or biomaterials, may be yet to be performed. Nevertheless, many biomedical areas could take advantage of NLs for in vivo studies. For example, supramolecular gels stabilized by nucleotide lipid salts, as described above, which are stabilized thanks to inter nucleobases interactions, represent one of the first examples of lipid based gels for the sustained release of drugs and/or biologics in vivo. ${ }^{[44]}$ Interestingly, NLs were also investigated as building blocks for the synthesis of ${ }^{99 \mathrm{~m}} \mathrm{Tc}$-labeled nanoparticles functionalized with a targeting moiety. ${ }^{[49]}$ This study showed that NL-based nanoparticles improved the pharmacokinetic profile of cisplatin (enhanced the half-life of cisplatin in vivo). Additionally, these nanoparticles were found to accumulate in the tumors of xenografted mice. These examples and the low toxicity of NLs (adverse effect level higher than $30 \mathrm{mg} / \mathrm{Kg} / \mathrm{Day}$ for nucleotide lipids not observed) highlight the strong potential of NLs for in vivo biomedical applications.

\section{Glyconucleolipids}

As its name implies, a glyconucleolipid (GNL) results from the covalent association of a lipid, a nucleoside and a sugar. Such synthetic bioinspired hybrid amphiphiles were first reported in 
the literature in 2005 (Figure 17). ${ }^{[50]}$ This original molecular structure, designed by Barthélémy and coll., was a glycosyl dicarbamate uridine $\mathbf{2 8}$ obtained in four steps from 5'dimethoxytrityluridine (Figure 17A). This very first synthesis involved cumbersome reactions requiring anhydrous conditions to give intermediates in moderate yields (from 36 to $55 \%$ according to the first three steps). They have demonstrated that these glyconucleosidebased amphiphilic structures could complex with DNA and form stable nucleic acid supramolecular assemblies. Through hyperchromicity studies, they showed a positive shift of thermal denaturation with an increasing amount of GNL (up to $3.5^{\circ} \mathrm{C}$ with 10 equivalents, Figure 17B). They also highlighted the complexation of phosphate anions by both amphiphilic structures and glucose moieties $\left({ }^{31} \mathrm{P}\right.$ NMR analysis), while DNA bases were stabilized via base pairing with nucleobases (Fourier transform spectroscopy).

The whole GNL structure was then involved in DNA complexation and stabilization according to a molecular model, as represented in Figure 17C.

Barthélémy's team delved into GNL systems in depth and developed an exclusive and extensive expertise regarding the synthesis and physico-chemical properties of these particular structures. Later, more complex GNLs, in which units are linked to each other by triazole moieties, were designed using CuAAC. ${ }^{[51]}$ This "click chemistry" is indeed more user-friendly and biocompatible than the former chemical reactions. Over the years, a series of GNLs have been synthesized with various chemical structures according to the nucleobase (thymidine, uridine), the lipidic (single or double chain, fluorocarbon chain, etc.) and the sugar (glucose, galactose, lactose) moieties. ${ }^{[52-57]}$ Different GNL topologies were also considered. Indeed, the sugar moiety was either linked to the nucleobase or the ribose $\mathrm{OH}-3^{\prime}$ position, allowing in this latter case potential base pairing ability. ${ }^{[58]}$ However, this review will first focus solely on thymidine-based GNLs featuring a hydrophobic chain on the 5'- 
ribose position (Figure 18A) and then on the more recently designed glyconucleoside bolaamphiphile (GNBA) ${ }^{[59,60]}$ (Figure 18B).

In 2009, Barthélémy and coll. designed a thymidine-based GNL bearing a fluorocarbon chain called glycosyl nucleoside fluorolipid, or GNF, using a double "click chemistry" strategy (Figure 19) ${ }^{[56]}$ After azidation on the thymidine 5'-position using classical conditions, ${ }^{[61]}$ compound 29 was submitted to the first click reaction, thus allowing the formation of the corresponding NL 30 with $64 \%$ yield. Finally, $N-3$ alkylation of thymidine with propargyl bromide provided the desired GNF $\mathbf{3 1}$ by means of a second click reaction with the suitable azido sugar.

Thanks to its amphiphilic nature, GNF exhibits interesting supramolecular properties in solvents. For example, GNF allows for the stabilization of gels in aqueous media thanks to intermolecular weak interactions such as hydrogen bonding, $\pi$ - $\pi$ stacking and hydrophobic effects. This gelation phenomenon showed promising results in several applications from tissue engineering to nanoparticle decontamination, as presented below.

Chassande and coll. demonstrated in 2012 that GNF-based hydrogels could be used as scaffolds for bone tissue engineering (Figure 20A). ${ }^{[62]}$ They described the formation of a weak hydrogel and its behavior toward cells and tissues. Rheological properties were fully compatible with cell culture since gelification occurred within 20 minutes at $37^{\circ} \mathrm{C}$ after heating at $65^{\circ} \mathrm{C}$ (Figure 20A top). The hydrogel cytocompatibility was demonstrated on metabolic activity (MTT assay) and viability (red neutral assay) of adipose stroma cells (ASCs). Thus, thanks to its rheological properties, the material was injected subcutaneously in mice in its liquid state via a syringe and reached its gel form in a few minutes at the injection area. After 60 days, a fibrous tissue containing blood vessels had invaded the hydrogel, confirming the creation of a vascularized microenvironment (Figure 20A bottom). Even if the system was non-compatible with isolated ASC cultures, it supported the survival of ASC 
aggregates and even promoted in vivo the differentiation of ASCs into functional osteoblasts without any osteogenic factors. These experiments highlighted GNF-based hydrogels as a promising tool for bone tissue engineering.

In 2014, Barthélémy's team reported the potential of GNF-based hydrogel as a trapping scaffold for nanoparticle decontamination (Figure 20B). ${ }^{[63]}$ For this purpose, the hydrogel was initially prepared with an aqueous phase contaminated with positively or negatively charged nanoparticles. After 2 to 48 hours of incubation, a decontaminated supernatant and a hydrogel with entrapped nanoparticles were observed. An example of QD decontamination is displayed in Figure 20B in a visible (Figure 20B top) and nanometric scale by TEM (Figure 20B bottom). These experiments clearly showed that nanoparticles initially present in the contaminated aqueous phase could be trapped in the GNF-based hydrogel, leaving the supernatant free of contaminants. These surprising decontamination properties could be explained by a thermodynamically favorable evolution of the system due to nanoparticle interactions with the supramolecular self-assembly.

In 2017, GNF 31 was used to design a gold electrode coated by the hydrogel, giving rise to a hybrid implantable macroporous device (Figure 20C). ${ }^{[64]}$ A coating of approximately 1-2 $\mu \mathrm{m}$, as evidenced by electron microscopy studies (SEM), was performed by simply dipping the electrode for a few hours in the hydrogel (Figure 20C top). This coating did not hinder the electrochemical behavior of the electrode considering the low difference between cyclic voltammograms with and without the hydrogel (Figure 20C bottom). The presence of GNFbased hydrogel as a coating, combined with its excellent biocompatibility, constitutes a good candidate for implantable electrochemical devices.

Considering the high interest of such innovative structures, Barthélémy's team synthesized a new GNL structure for drug delivery applications in 2011 (Figure 21). ${ }^{[57]}$ Following the same double-click chemistry approach, a thymidine-based GNL bearing a double lipid chain $\mathbf{3 2}$ was 
synthesized (Figure 21A). In contrast to its GNF homolog, 32 did not induce the formation of a hydrogel. This double-chain GNL 32 induced the formation of liposome-like structures in the presence of lecithin with carbohydrate moieties at their surface. It was hypothesized that such liposomes featuring sugar moieties at the surfaces could favor interactions with cell membranes (Figure 21B). GNL-based and glycolipid (GL)-based liposomes labeled with a fluorescent dye were incubated with stem cells (ASCs) for 48 hours. Unexpectedly, confocal microscopy experiments showed that only the formulations containing the GNL 32 were uptaken into the cytoplasm, highlighting two major results. First, it proved that these structures displayed carbohydrate moieties at the liposome outer surface, allowing interactions with cell membranes that favored their internalization (Figure $21 \mathrm{C}$ left). Second, the poor internalization of GL-based formulations into the cells (ASCs) showed that the nucleoside also played a crucial role in this process (Figure $21 \mathrm{C}$ right). This could be explained by the high contribution of this moiety in terms of weak interactions as hydrogen bonding or $\pi-\pi$ stacking. This study clearly showed that these structures are fully suitable for cell-targeting systems.

The same year, Lindhorst and coll. designed glycothymidines as in situ photoswitchable compounds to study structural changes (orientation, accessibility) of surface-exposed carbohydrates within the glycocalyx (Figure 22). ${ }^{[65]}$ For this purpose, amphiphilic glycothymidines bearing a sugar at the $N-3$ position and an alkyl chain at the secondary alcohol 5'-position were synthesized following a six step-sequence (Figure 22A top). 33 was submitted to a photochemical [2+2] cycloaddition under UV light $(>295 \mathrm{~nm}$ ), providing dimers 34 (Figure 22A bottom) whose structures were evidenced by changes in UV spectra and anomeric ribose $\mathrm{H} 1$ ' peaks shifts in ${ }^{1} \mathrm{H}$ NMR. To perform in situ photodimerization into a synthetic in vivo glycosylated cell surface model, they used SDS micelles in which amphiphilic glycothymidines were incorporated as monomers. Calculation of diffusion 
constants by pulsed field gradient (PGG) ${ }^{1} \mathrm{H}$ NMR experiments allowed them to demonstrate the proper incorporation of $\mathbf{3 3}$ into SDS micelles. They also demonstrated the surface-exposed orientation of the sugar on the monomer $\mathbf{3 3}$ while alkyl chains were rather embedded into SDS micelles (Figure 22B top). In situ photodimerization could be successfully achieved in a supramolecular model and demonstrated due to the appearance of multiple characteristic anomeric ribose protons $\mathrm{H} 1$ '. In situ [2+2] cycloaddition of these glycomimetics into a glycocalyx model altered the pattern of surface exposed carbohydrates, thus providing the first insights on the role of conformational control in glycobiology.

More recently, Barthélémy's team designed a new GNL structure called GNBA, or glyconucleoside bolaamphiphile, which gathers two glyconucleoside units (polar heads) linked by a lipidic chain (hydrophobic tail). The synthetic route, using the same double "click chemistry" approach, is strictly identical to the regular GNL synthesis. To form this specific bola form, the last click reaction step involves two equivalents of $\mathbf{3 5}$ for one equivalent of diacetylenic lipid moiety. ${ }^{[56,59]}$

Very interestingly, bola compound $\mathbf{3 6}$ allowed the formation of a low molecular weight hydrogel (Figure 23A). TEM images revealed the presence of a dense anisotropic fibrillar network (Figure 23B), different from GNF-based hydrogel 31, where a less connected network was observed. Thus, GNBA-based hydrogel displayed improved rheological properties compared to GNF-based hydrogel. At a fixed angular frequency of $1 \mathrm{rad} . \mathrm{s}^{-1}$, its G' was almost twenty times higher than GNF-based hydrogel $G^{\prime}$ at the same molar concentration. This enhanced stiffness had a direct effect on cell development as it could promote mechanotransduction (conversion of a mechanical stimulus into electrochemical activity). For biomedical purposes, injectable systems have a highly valuable advantage, thereby avoiding implantation. GNBA-based hydrogel 36 showed thixotropic properties measured by rheology through several cycles of high (Figure 23C, 2 i.e., liquefaction) and 
low strains (Figure 23C, $1+3$ i.e., gel state). This has been put into practice with a subcutaneous injection on a mouse. Two hours after injection, a biopsy showed the presence of the hydrogel (ovoid shape) at the injection area, proving its injectability. GNBA-based hydrogels also displayed cytocompatibility and non-cytotoxicity with ASC cells evaluated by MTT assays. In contrast to GNF 31, GNBA-based hydrogel 36 allowed the culture of isolated stem cells. Confocal microscopy coupled to live/dead viability assays displayed cell adhesion, gave evidence regarding fibroblastoid phenotypes on almost all cells, and some cell divisions (Figure 23D). This result was correlated with enhanced rheological properties displayed by GNBA versus GNF. All these experiments note the control of stem cell behavior by low molecular weight hydrogel architectures and the use of GNBA-based hydrogel as an extracellular matrix substitute.

\section{Responsive materials}

Interest in responsive materials has continued over several decades, and many investigations have been dedicated to developing sensitive molecules or macromolecules that can be inserted into smart materials or devices. The vast majority of publications describe stimuli-responsive polymers ${ }^{[66]}$ sensitive to different stimuli, including changes in $\mathrm{pH},{ }^{[67,68]}$ light,${ }^{[69,70]}$ redox, ${ }^{[71,72]}$ enzymes, ${ }^{[73]}$ salt concentration, temperature, ${ }^{[74]}$ ultrasound, ${ }^{[75]}$ and electric ${ }^{[76]}$ or magnetic ${ }^{[77]}$ stimulation. In parallel, supramolecular systems ${ }^{[78]}$ are emerging as a clear opportunity in biomedical fields thanks to their specificity and ability to respond to stimuli found in living systems. In this section, we will highlight recent results and future trends that exploit NLs and GNLs. It is our goal to emphasize these non-polymeric molecules as far as novel applications and new generations of smart materials can be achieved. Biomaterial construction using supramolecular principles via a bottom-up approach enables the molecular modulation of 
chemical, mechanical, and biological properties. Interestingly, the dynamic character of supramolecular interactions provides systems that can respond to specific stimuli through a fundamental change in material properties. The ability to accurately control structure, bioactivity, and mechanical properties using a variety of biologically compatible triggers represents an obvious option for controlling the delivery of therapeutics or drugs. Depending on the applications, reversible or irreversible switches may also be envisioned. As shown in Figure 24, two types of stimuli, i.e., externally (temperature, ultrasound, light, magnetic field, mechanical stress, etc.) and internally ( $\mathrm{pH}$, specific, molecular interactions, enzymes, salts, ionic strength, redox potential, etc.) applied triggers, may be developed in the case supramolecular systems. Regarding drug delivery applications, both internal and external stimuli have been investigated in the case of NLs and GNLs, particularly redox, $\mathrm{pH}$, salt concentration and mechanical stress (Figure 24 gray frames).

\subsection{Internal or environmental stimuli}

Internal stimuli, which correspond to local or environmental changes in terms of $\mathrm{pH}$, ionic strength, or redox potential, are also common triggers used for the release of active principle ingredients (APIs) from supramolecular systems. ${ }^{[74,79]}$ A basic principle is to induce changes in a supramolecular system upon stimulation via a selected trigger. Thus, the material undergoes a transition from one organization to another, resulting in the release of APIs.

Regarding salt concentration stimulus, Barthélémy’s strategy entailed using calcium cations associated with phosphate moieties belonging to both nucleotide lipids and DNA. ${ }^{[40]}$ In addition to hydrogen-bonding and base $\pi-\pi$ stacking, such a cationic bridge between the two species would allow the stabilization of complexes. It was demonstrated that the anionic nucleotide lipid $\left(\mathrm{DiC}_{16} \mathrm{dT}\right)$ was able to bind nucleic acids via DNA-nucleotide lipid 
interactions, allowing the stabilization of DNA plasmid lipoplexes in the presence of $\mathrm{Ca}^{2+}$. To use these lipoplexes as an alternative to cationic systems for transfection applications, they hypothesized that the complex stability could be tuned depending on the $\mathrm{Ca}^{2+}$ concentration. It was observed that the nucleotide lipid/DNA stability depended on $\mathrm{Ca}^{2+}$ concentration. Indeed, nucleotide lipid/DNA complexes were stable only above a concentration of $1 \mathrm{mM}$, which is much higher than the intracellular $\mathrm{Ca}^{2+}$ concentration $\left(\left[\mathrm{Ca}^{2+}\right]=1 \mu \mathrm{M}\right)$, indicating that lipoplexes could release DNA into cells. The results of transfection assays performed on mammalian cell lines (Hek 293 human embryo kidney) showed that anionic nucleotide lipids were more efficient compared to regular anionic phospholipids in the same conditions. This demonstrated that the nucleotide moiety influenced lipid-DNA associations and enhanced transfection efficacy of the natural anionic phospholipids.

The site-specific release of APIs represents a promising approach to address issues in drug delivery. ${ }^{[80]}$ In that regard, $\mathrm{pH}$-sensitive drug delivery systems have been widely investigated. For example, it is known that a $\mathrm{pH}$ sensitive system can take advantage of the acidic $\mathrm{pH}$ of the tumor environment. ${ }^{[81]}$ Additionally, large $\mathrm{pH}$ shifts from neutral $\mathrm{pH}$ in blood to $\mathrm{pH}=4.0-6.5$ in intracellular endosomes can be used as a trigger to favor intracellular drug delivery. ${ }^{[82-85]}$ $\mathrm{pH}$ responsive NLs were reported only recently (Figure 25). ${ }^{[86]}$ In this work, a novel approach involving orthoester-based NLs was developed in order to control fusion properties. It was demonstrated that orthoester modification of NLs (ONLs) resulted in the formation of $\mathrm{pH}-$ sensitive liposomes able to release fatty alcohols into biological membranes in acidic conditions. The presence of fatty alcohols destabilized vesicles, and the formation of lamellar phases was observed (Figure 25A, Figure 25B). The association of fatty alcohol with phosphocholine natural lipids (DOPC) could explain such a transition via a modulation of the membrane curvature. As an example of an application, a cationic ONL was used to deliver a 
therapeutic siRNA targeting particles of a human RecQ helicase (RECQL4) overexpressed in aggressive breast cancers (Luminal B).

As an alternative to $\mathrm{pH}$ responsiveness, several cleavable or detachable chemical systems have been investigated, including those sensitive to reducing conditions. ${ }^{[87,88]}$ Indeed, the tumoral extracellular microenvironment is known to be reductive due to the release of glutathione and reductases by tumor cells and the presence of redox enzymes. Within this context, a nucleoside lipid featuring a cleavable disulfide polyethylene glycol (PEG) was synthesized in order to improve the internalization of drug carrier into tumor cells. PEGylation of nano-objects remains an important technology, in particular for in vivo drug delivery. However, PEGylation is detrimental to cellular internalization of stealth nanoobjects due to their poor interactions with cellular membranes. An alternative is to release PEG moieties next to tumor cells in order to restore cellular internalization. Barthélémy and coll. reported a PEG detachable nucleotide lipid named DOU-SS-PEG2000 (Figure 26A). ${ }^{[89]}$ This nucleoside lipid self-organized to spontaneously yield micelles $20 \mathrm{~nm}$ in diameter. Physico-chemical studies revealed that a reduction induced a transition from micellar systems to vesicular states (Figure 26B). In the case of liposome formulations with a cationic lipid (DOTAU), cleavage of PEG in reduction conditions resulted in liposomes featuring cationic surfaces (Figure 26C). As a result, an enhanced cellular internalization of micelles and liposomes nano-objects in ovarian cancer cells line was observed. This demonstrates that PEG detachable nucleoside lipids can be inserted in formulations to optimize cellular internalization efficacy for in vivo anticancer applications.

\subsection{External stimuli}

Numerous responsive drug delivery systems based on externally applied triggers such as light, temperature, ultrasound, electric or magnetic fields have been investigated. ${ }^{[90,91]}$ With regard 
to mechanically responsive materials, we are currently witnessing an increasing number of reports, particularly in the context of biomedicine, which highlights the relevance of dynamic mechanical properties in bioengineering ${ }^{[2,93]}$ and drug delivery applications ${ }^{[94,95]}$. While recognizing the importance of nucleobase interactions in self-assembly formation and in an effort to expand the synthetic mechanically responsive supramolecular toolbox, a GNL was studied by Grinstaff and coll. as a responsive low molecular weight gel for drug delivery applications (Figure 27). ${ }^{[96]}$ As expected, a weak mechanical shear stimulation applied to the GNL-based hydrogel previously loaded with an antibody (anti-TNF $\alpha$ ) increased the release of this when compared with the non-stimulated hydrogel. This demonstrates the utility of such bioinspired materials for the controlled release of delicate and bioactive substances.

The use of NLs or GNLs for preparing responsive supramolecular materials, which has been investigated only recently, represents a unique approach that complements previous systems based on polymers. Continued research in this field will afford novel responsive supramolecular materials (gels, nanoparticles, micro- and nano-emulsions) able to respond to different stimuli, including magnetic and electrical fields, light, ultrasound, enzymes or specific molecular interactions, which are still unexplored so far (Figure 24). Work is currently in progress on a number of bioinspired nucleoside structures to extend the current repertoire of responsive systems.

\section{Conclusion}

Recent publications in the field of nucleic acid-based amphiphiles over the past seven years open up promising avenues for the design and development of soft materials. Nature is an obvious source of inspiration, and looking at the supramolecular behavior of biomolecules could help us to discover new advanced materials. The above examples illustrate how 
efficient supramolecular devices, biotechnological tools, drug delivery systems, therapeutic strategies and responsive materials have been devised from nucleolipids and glyconucleolipids. By examining the scientific contribution of main academic actors in the field, we have identified two areas of interest for the next decade: a) a fundamental exploration of new methods to construct soft materials (e.g., low molecular weight gels, nanoobjects, responsive devices) and b) the evaluation of these supramolecular tools for biomedical and technological applications (e.g., regenerative medicine, bioprinting, drug delivery, medicinal chemistry).

Regarding the fundamental aspects of nucleolipids as supramolecular tools, one will note that guanine and cytosine derivatives have been poorly investigated compared to thymine, adenine, or uracil structures. Interestingly, guanine and cytosine are known to form particular supramolecular structures in living systems. G-quadruplexes, formed by nucleic acids rich in guanine, correspond to four-stranded helical secondary structures located most of the time at the end of chromosomes. Such supramolecular organizations, which would be involved in important mechanisms for regulating multiple biological processes in vivo, ${ }^{[97]}$ are stabilized by the presence of cations, especially potassium. Likewise, i-motifs, ${ }^{[98]}$ which can be observed in nucleic acid sequences rich in cytosine, correspond to four-stranded oligonucleotide supramolecular structures. Those secondary organizations are stabilized by acidic conditions. Thanks to their $\mathrm{pH}$-dependent folding, i-motifs would be of interest as $\mathrm{pH}$ switches for applications in nanotechnology. Hence, the properties of G-quadruplexes and i-motifs hold great potential for the development of sensors or switches, which could be useful for advanced biomedical applications. The synthetic access to guanine and cytosine nucleolipids has consistently lagged behind that of thymine, adenine and uracil analogs. As a consequence, many key supramolecular developments in the field have been limited to thymine-thymine, adenine-adenine or thymine-adenine systems. Extending the nucleolipids repertoire to at least 
the five natural nucleobase derivatives (A, T, C, G, U) would open the way toward programmable or encoded supramolecular systems and, for example, allow the construction of G-quadruplex or i-motif responsive devices.

The covalent combination of nucleic acid features with lipids provides means and tools to construct complex matter possessing emerging features that do not exist when these families are taken separately. Through the appropriate supramolecular organization of specific nucleolipids in organized patterns, they may be amenable to the construction of novel smart and functional materials for biomedical applications. For example, bearing in mind the first results obtained in the field of biomaterials, one can easily anticipate that nucleolipid-based supramolecular soft materials should play a major role in the field of regenerative medicine, tissue engineering, bioprinting or cellular therapy. Indeed, it is known that mechanotransduction between cells and the extracellular matrix strongly impacts on cellular functions and fate. ${ }^{[99]}$ The design of 3D soft materials using nucleic acid based amphiphiles offers a full control of many chemical and physicochemical parameters, which would be of high interest in these fields. Such accurately engineered 3D materials should pave the way for investigating the mechanotransduction of multicellular aggregates under controlled chemical and mechanical parameters.

Together with the corresponding areas in chemistry, biology, physics and technology, future studies with these hybrid biomolecules will certainly open a door to the design of both simple biocompatible devices or complex and programmable, encoded, supramolecular materials.

\section{Acknowledgements}

This work, realized within the framework of the Laboratory of Excellence AMADEus with the reference ANR-10-LABX-0042-AMADEUS, has benefitted from aid by the state operated "Agence Nationale de la Recherche" under the program "Initiative for Excellence IdEx 
Bordeaux" with the reference ANR-10-IDEX-0003-02. The work has also been supported by INSERM and CNRS. Julie Baillet and Aladin Hamoud thank respectively DGA and the French MESRI for Ph.D. grants.

\section{References}

[1] H. Rosemeyer, Chem. Biodiversity 2005, 2, 977.

[2] A. Gissot, M. Camplo, M. W. Grinstaff, P. Barthélémy, Org. Biomol. Chem. 2008, 6, 1324.

[3] U. Pradere, E. C. Garnier-Amblard, S. J. Coats, F. Amblard, R. F. Schinazi, Chem. Rev. 2014, $114,9154$.

[4] B. Roy, A. Depaix, C. Périgaud, S. Peyrottes, Chem. Rev. 2016, 116, 7854.

[5] A. Patwa, A. Gissot, I. Bestel, P. Barthélémy, Chem. Soc. Rev. 2011, 40, 5844.

[6] M. Raouane, D. Desmaële, G. Urbinati, L. Massaad-Massade, P. Couvreur, Bioconjugate Chem. 2012, 23, 1091.

[7] A. Gissot, K. Oumzil, A. Patwa, P. Barthélémy, New J. Chem. 2014, 38, 5129.

[8] L. Simeone, G. Mangiapia, C. Irace, A. Di Pascale, A. Colonna, O. Ortona, L. De Napoli, D. Montesarchio, L. Paduano, Mol. Biosyst. 2011, 7, 3075.

[9] L. Simeone, G. Mangiapia, G. Vitiello, C. Irace, A. Colonna, O. Ortona, D. Montesarchio, L. Paduano, Bioconjugate Chem. 2012, 23, 758.

[10] S. Leijen, S. A. Burgers, P. Baas, D. Pluim, M. Tibben, E. van Werkhoven, E. Alessio, G. Sava, J. H. Beijnen, J. H. M. Schellens, Invest. New Drugs 2015, 33, 201.

[11] G. Mangiapia, G. D’Errico, L. Simeone, C. Irace, A. Radulescu, A. Di Pascale, A. Colonna, D. Montesarchio, L. Paduano, Biomaterials 2012, 33, 3770. 
[12] D. Montesarchio, G. Mangiapia, G. Vitiello, D. Musumeci, C. Irace, R. Santamaria, G. D’Errico, L. Paduano, Dalton Trans. 2013, 42, 16697.

[13] L. Simeone, C. Irace, A. Di Pascale, D. Ciccarelli, G. D’Errico, D. Montesarchio, Eur. J. Med. Chem. 2012, 57, 429.

[14] C. Riccardi, D. Musumeci, C. Irace, L. Paduano, D. Montesarchio, Eur. J. Org. Chem. 2017, 2017, 1100 .

[15] X. Mulet, T. Kaasgaard, C. E. Conn, L. J. Waddington, D. F. Kennedy, A. Weerawardena, C. J. Drummond, Langmuir 2010, 26, 18415.

[16] O. Kaczmarek, H. A. Scheidt, A. Bunge, D. Föse, S. Karsten, A. Arbuzova, D. Huster, J. Liebscher, Eur. J. Org. Chem. 2010, 2010, 1579.

[17] A. Nuthanakanti, S. G. Srivatsan, ACS Appl. Mater. Interfaces 2017, 9, 22864.

[18] E. Malecki, H. Rosemeyer, Helv. Chim. Acta 2010, 93, 1500.

[19] L. Moreau, M. Camplo, M. Wathier, N. Taib, M. Laguerre, I. Bestel, M. W. Grinstaff, P. Barthélémy, J. Am. Chem. Soc. 2008, 130, 14454.

[20] N. Taib, A. Aimé, L. Moreau, M. Camplo, S. Houmadi, B. Desbat, M. Laguerre, M. W. Grinstaff, I. Bestel, P. Barthélémy, J. Colloid Interface Sci. 2012, 377, 122.

[21] E. Malecki, C. Knies, E. Werz, H. Rosemeyer, Chem. Biodiversity 2013, 10, 2209.

[22] K. Köstler, E. Werz, E. Malecki, M. Montilla-Martinez, H. Rosemeyer, Chem. Biodiversity 2013, 10, 39.

[23] E. Malecki, R. Viere, H. Rosemeyer, Chem. Biodiversity 2013, 10, 1828.

[24] A. Farhat, E. Malecki, G. A. Bonaterra, D. Röthlein, M. Wolf, J. Schmitt, H. Rosemeyer, R. Kinscherf, Chem. Biodiversity 2014, 11, 469.

[25] E. Malecki, A. Farhat, G. A. Bonaterra, D. Röthlein, M. Wolf, J. Schmitt, R. Kinscherf, H. Rosemeyer, Chem. Biodiversity 2013, 10, 2235.

[26] A. A. Tanpure, S. G. Srivatsan, Chem. - Eur. J. 2011, 17, 12820. 
[27] M. G. Pawar, S. G. Srivatsan, Org. Lett. 2011, 13, 1114.

[28] A. Nuthanakanti, S. G. Srivatsan, Nanoscale 2016, 8, 3607.

[29] C. Montis, S. Milani, D. Berti, P. Baglioni, J. Colloid Interface Sci. 2012, 373, 57.

[30] M. Banchelli, D. Berti, P. Baglioni, Angew. Chem. Int. Ed. 2007, 46, 3070.

[31] S. Milani, F. Baldelli Bombelli, D. Berti, P. Baglioni, J. Am. Chem. Soc. 2007, 129, 11664.

[32] C. Montis, Y. Gerelli, G. Fragneto, T. Nylander, P. Baglioni, D. Berti, Colloids Surf., B. 2016, 137, 203.

[33] M. Y. Arteta, D. Berti, C. Montis, R. A. Campbell, L. A. Clifton, M. W. A. Skoda, O. Soltwedel, P. Baglioni, T. Nylander, Soft Matter 2014, 10, 8401.

[34] M. Y. Arteta, D. Berti, C. Montis, R. A. Campbell, C. Eriksson, L. A. Clifton, M. W. A. Skoda, O. Soltwedel, A. Koutsioubas, P. Baglioni, T. Nylander, Soft Matter 2015, 11, 1973.

[35] I. Tsybulskaya, T. Kulak, A. Baranovsky, M. Golubeva, B. Kuzmitsky, E. Kalinichenko, Bioorg. Med. Chem. 2013, 21, 5414.

[36] P. Sandin, F. B. Bombelli, B. Castroflorio, C. Müller, J. Obermeier, G. Karlsson, K. Edwards, P. Baglioni, D. Berti, New J. Chem. 2014, 38, 5247.

[37] K. L. Gangadhara, P. Srivastava, J. Rozenski, H.-P. Mattelaer, V. Leen, W. Dehaen, J. Hofkens, E. Lescrinier, P. Herdewijn, J. Syst. Chem. 2014, 5, 5.

[38] N. Campins, P. Dieudonné, M. W. Grinstaff, P. Barthélémy, New J. Chem. 2007, 31, 1928.

[39] I. Bestel, N. Campins, A. Marchenko, D. Fichou, M. W. Grinstaff, P. Barthélémy, J. Colloid Interface Sci. 2008, 323, 435.

[40] S. Khiati, N. Pierre, S. Andriamanarivo, M. W. Grinstaff, N. Arazam, F. Nallet, L. Navailles, P. Barthélémy, Bioconjugate Chem. 2009, 20, 1765. 
[41] R. Iwaura, K. Yoshida, M. Masuda, K. Yase, T. Shimizu, Chem. Mater. 2002, 14, 3047.

[42] B. Desbat, N. Arazam, S. Khiati, G. Tonelli, W. Neri, P. Barthélémy, L. Navailles, Langmuir 2012, 28, 6816.

[43] G. Tonelli, K. Oumzil, F. Nallet, C. Gaillard, L. Navailles, P. Barthélémy, Langmuir 2013, 29, 5547.

[44] M. A. Ramin, K. R. Sindhu, A. Appavoo, K. Oumzil, M. W. Grinstaff, O. Chassande, P. Barthélémy, Adv. Mater. 2017, 29, 1605227.

[45] K. Sugiyasu, M. Numata, N. Fujita, S. M. Park, Y. J. Yun, B. H. Kim, S. Shinkai, Chem. Commun. 2004, 0, 1996.

[46] A. Patwa, G. Salgado, F. Dole, L. Navailles, P. Barthélémy, Org. Biomol. Chem. 2013, 11,7108 .

[47] S. Khiati, D. Luvino, K. Oumzil, B. Chauffert, M. Camplo, P. Barthélémy, ACS Nano 2011, 5,8649 .

[48] K. Oumzil, M. A. Ramin, C. Lorenzato, A. Hémadou, J. Laroche, M. J. Jacobin-Valat, S. Mornet, C.-E. Roy, T. Kauss, K. Gaudin, G. Clofent-Sanchez, P. Barthélémy, Bioconjugate Chem. 2016, 27, 569.

[49] K. Oumzil, S. Khiati, M. Camplo, M. Koquely, K. Chuttani, S. Chaturvedi, A. K. Mishra, P. Barthélémy, New J. Chem. 2014, 38, 5240.

[50] J. Arigon, C. A. H. Prata, M. W. Grinstaff, P. Barthélémy, Bioconjugate Chem. 2005, 16,864 .

[51] H. C. Kolb, M. G. Finn, K. B. Sharpless, Angew. Chem. Int. Ed. 2001, 40, 2004.

[52] L. Latxague, M.-J. Dalila, A. Patwa, S. Ziane, O. Chassande, G. Godeau, P. Barthélémy, C. R. Chim. 2012, 15, 29.

[53] M. A. Ramin, J. Baillet, S. Benizri, L. Latxague, P. Barthélémy, New J. Chem. 2016, 40, 9903. 
[54] G. Godeau, J. Bernard, C. Staedel, P. Barthélémy, Chem. Commun. 2009, 0, 5127.

[55] G. Godeau, P. Barthélémy, Langmuir 2009, 25, 8447.

[56] G. Godeau, C. Brun, H. Arnion, C. Staedel, P. Barthélémy, Tetrahedron Lett. 2010, 51, 1012.

[57] L. Latxague, S. Ziane, O. Chassande, A. Patwa, M.-J. Dalila, P. Barthélémy, Chem. Commun. 2011, 47, 12598.

[58] L. Latxague, A. Patwa, E. Amigues, P. Barthélémy, Molecules 2013, 18, 12241.

[59] L. Latxague, M. A. Ramin, A. Appavoo, P. Berto, M. Maisani, C. Ehret, O. Chassande, P. Barthélémy, Angew. Chem. Int. Ed. 2015, 54, 4517.

[60] L. Latxague, A. Gaubert, D. Maleville, J. Baillet, M. A. Ramin, P. Barthélémy, Gels 2016, 2,25 .

[61] X. Hu, M. T. Tierney, M. W. Grinstaff, Bioconjugate Chem. 2002, 13, 83.

[62] S. Ziane, S. Schlaubitz, S. Miraux, A. Patwa, C. Lalande, I. Bilem, S. Lepreux, B. Rousseau, J.-F. Le Meins, L. Latxague, P. Barthélémy, O. Chassande, Eur. Cells Mater. 2012, 23, 147.

[63] A. Patwa, J. Labille, J.-Y. Bottero, A. Thiéry, P. Barthélémy, Chem. Commun. 2015, 51, 2547.

[64] D. Jain, A. Karajic, M. Murawska, B. Goudeau, S. Bichon, S. Gounel, N. Mano, A. Kuhn, P. Barthélémy, ACS Appl. Mater. Interfaces 2017, 9, 1093.

[65] K. Schwekendiek, H. Kobarg, L. Daumlechner, F. D. Sönnichsen, T. K. Lindhorst, Chem. Commun. 2011, 47, 9399.

[66] D. Roy, J. N. Cambre, B. S. Sumerlin, Prog. Polym. Sci. 2010, 35, 278.

[67] W. Gao, J. M. Chan, O. C. Farokhzad, Mol. Pharmaceutics 2010, 7, 1913.

[68] A. J. Convertine, C. Diab, M. Prieve, A. Paschal, A. S. Hoffman, P. H. Johnson, P. S. Stayton, Biomacromolecules 2010, 11, 2904. 
[69] J. Jiang, X. Tong, Y. Zhao, J. Am. Chem. Soc. 2005, 127, 8290.

[70] H. Kang, A. C. Trondoli, G. Zhu, Y. Chen, Y.-J. Chang, H. Liu, Y.-F. Huang, X. Zhang, W. Tan, ACS Nano 2011, 5, 5094.

[71] J. Xu, A. Singh, M. M. Amiji, BMC Cancer 2014, 14, 75.

[72] R. Cheng, F. Feng, F. Meng, C. Deng, J. Feijen, Z. Zhong, J. Controlled Release 2011, $152,2$.

[73] M. T. Basel, T. B. Shrestha, D. L. Troyer, S. H. Bossmann, ACS Nano 2011, 5, 2162.

[74] D. Schmaljohann, Adv. Drug Delivery Rev. 2006, 58, 1655.

[75] S. Hernot, A. L. Klibanov, Adv. Drug Delivery Rev. 2008, 60, 1153.

[76] J. Ge, E. Neofytou, T. J. Cahill, R. E. Beygui, R. N. Zare, ACS Nano 2012, 6, 227.

[77] R. Cheng, F. Meng, C. Deng, H.-A. Klok, Z. Zhong, Biomaterials 2013, 34, 3647.

[78] M. J. Webber, Bioeng. Transl. Med. 2016, 1, 252.

[79] S. Ganta, H. Devalapally, A. Shahiwala, M. Amiji, J. Controlled Release 2008, 126, 187.

[80] C. S. Kim, B. Duncan, B. Creran, V. M. Rotello, Nano Today 2013, 8, 439.

[81] I. F. Tannock, D. Rotin, Cancer Res. 1989, 49, 4373.

[82] C. L. Walsh, J. Nguyen, F. C. Szoka, Chem. Commun. 2012, 48, 5575.

[83] E. Fattal, P. Couvreur, C. Dubernet, Adv. Drug Delivery Rev. 2004, 56, 931.

[84] C. Masson, M. Garinot, N. Mignet, B. Wetzer, P. Mailhe, D. Scherman, M. Bessodes, J. Controlled Release 2004, 99, 423.

[85] Z. Huang, X. Guo, W. Li, J. A. MacKay, F. C. Szoka, J. Am. Chem. Soc. 2006, 128, 60.

[86] K. Oumzil, S. Benizri, G. Tonelli, C. Staedel, A. Appavoo, M. Chaffanet, L. Navailles, P. Barthélémy, ChemMedChem 2015, 10, 1797.

[87] D. Kirpotin, K. Hong, N. Mullah, D. Papahadjopoulos, S. Zalipsky, FEBS Lett 1996, 388, 115. 
[88] P. Sun, D. Zhou, Z. Gan, J. Controlled Release 2011, 155, 96.

[89] K. Oumzil, S. Khiati, M. W. Grinstaff, P. Barthélémy, J. Controlled Release 2011, 151, 123.

[90] R. Langer, Science 1990, 249, 1527.

[91] S. Mura, J. Nicolas, P. Couvreur, Nat. Mater. 2013, 12, 991.

[92] A. J. Engler, S. Sen, H. L. Sweeney, D. E. Discher, Cell 2006, 126, 677.

[93] J. H. Wen, L. G. Vincent, A. Fuhrmann, Y. S. Choi, K. C. Hribar, H. Taylor-Weiner, S. Chen, A. J. Engler, Nat. Mater. 2014, 13, 979.

[94] H. D. Lu, D. E. Soranno, C. B. Rodell, I. L. Kim, J. A. Burdick, Adv. Healthcare Mater. 2013, 2, 1028.

[95] N. Korin, M. Kanapathipillai, B. D. Matthews, M. Crescente, A. Brill, T. Mammoto, K. Ghosh, S. Jurek, S. A. Bencherif, D. Bhatta, A. U. Coskun, C. L. Feldman, D. D. Wagner, D. E. Ingber, Science 2012, 337, 738.

[96] J. A. Kaplan, P. Barthélémy, M. W. Grinstaff, Chem. Commun. 2016, 52, 5860.

[97] D. Rhodes, H. J. Lipps, Nucleic Acids Res. 2015, 43, 8627.

[98] H. A. Day, P. Pavlou, Z. A. E. Waller, Bioorg. Med. Chem. 2014, 22, 4407.

[99] Y. Zhang, K. Liao, C. Li, A. C. K. Lai, J.-J. Foo, V. Chan, Bioengineering 2017, 4, 72.

A

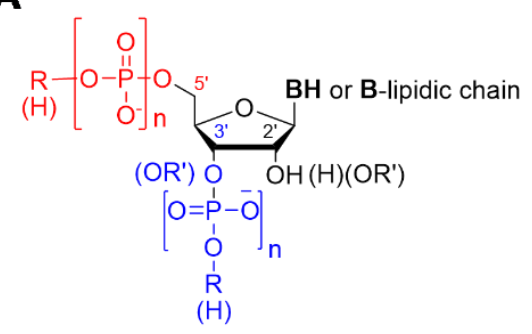

Nucleotide Lipid $n=1,2,3, \mathrm{R}$ or $\mathrm{R}^{\prime}=$ lipidic chain or lipidic chain on $\mathrm{B}$ Nucleoside Lipid $\mathrm{n}=0$ R or $\mathrm{R}^{\prime}=$ lipidic chain or lipidic chain on $\mathrm{B}$
B

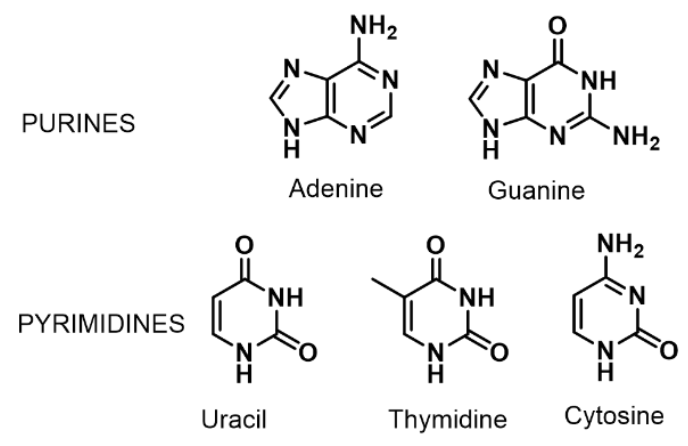


Figure 1. A. General structure of nucleolipids showing different possible substitutions on the ribose moieties at the 2'-, 3'- and 5'-positions. Nucleotide lipids possess a phosphate moiety, whereas nucleoside lipids are only functionalized with lipid chains. B. Chemical structures of purine and pyrimidine natural nucleobases.

A

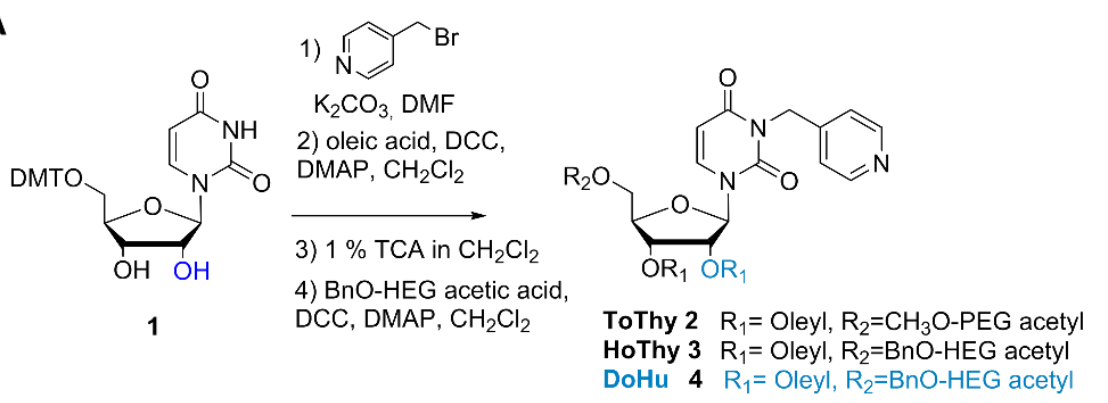

B
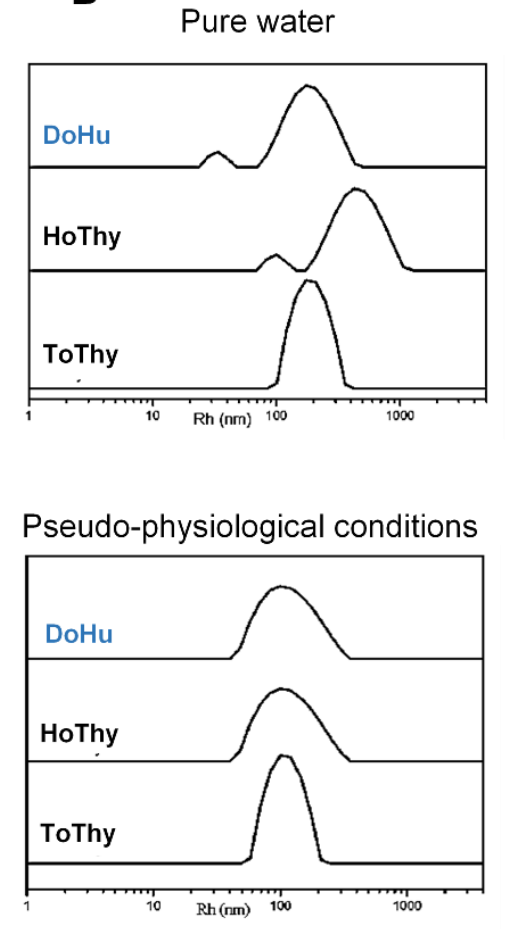
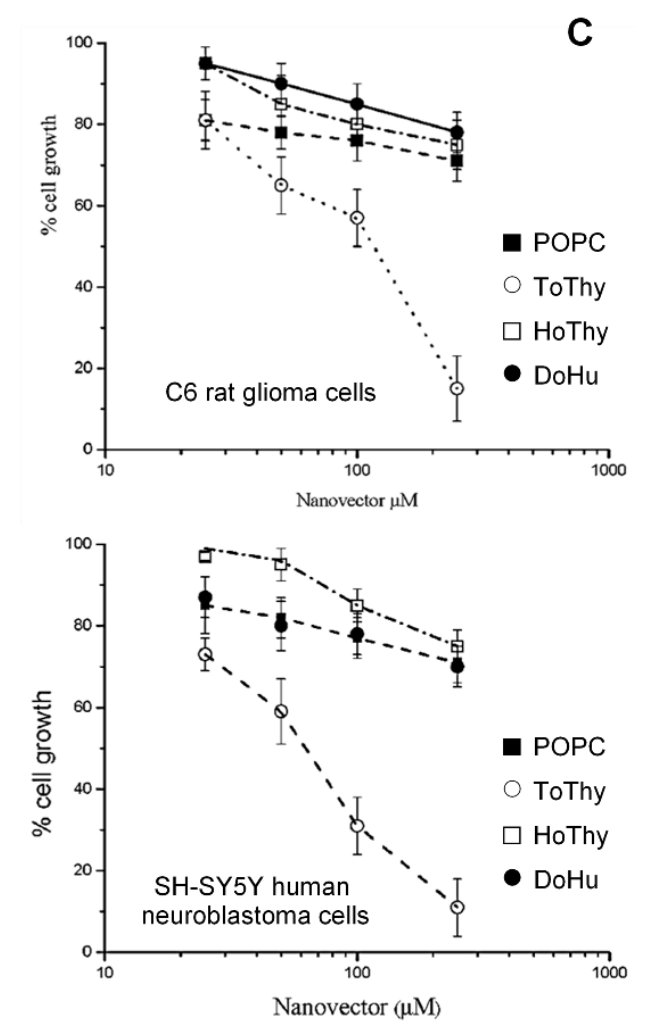

Figure 2. Nucleolipid nanovectors as molecular carriers. A. NLs synthetic route. B. Hydrodynamic radii distribution at $90^{\circ}$ of aggregates formed by NLs in both water (top) and pseudo-physiological conditions (bottom). Concentration of $0.2 \mathrm{mmol} . \mathrm{kg}^{-1}$ for all systems. C. Cell viability evaluations (MTT assays) performed on indicated cell lines treated for 72 hours with nanovectors. Reproduced with permission. ${ }^{[8]}$ Copyright 2011, Royal Society of Chemistry. 
A
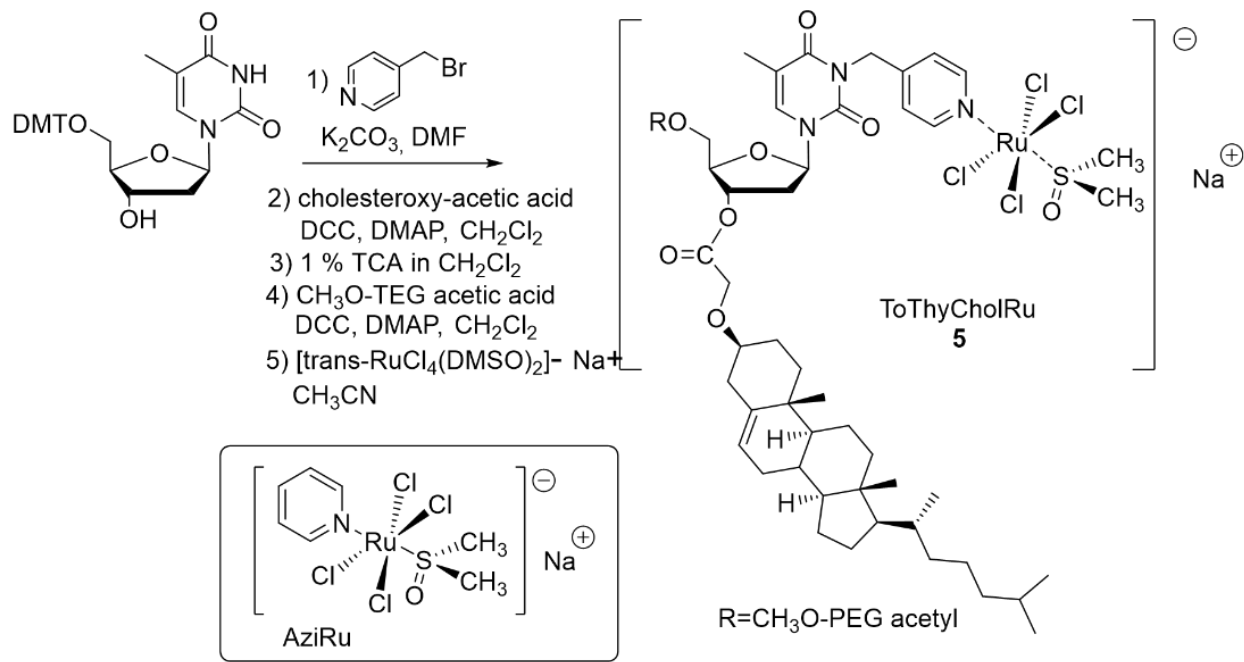

B

C
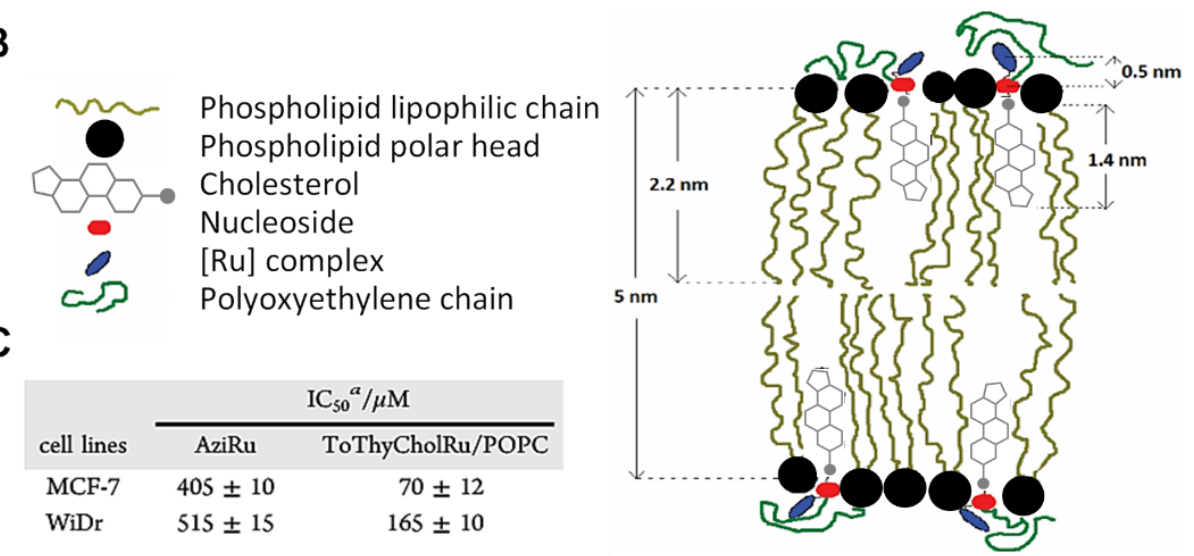

Figure 3. Cholesterol-based nucleolipid-ruthenium complex for antineoplastic therapy. A. Synthetic procedure for the preparation of ToThyCholRu (top) and molecular structure of pyridine-based ruthenium complex (frame). B. Schematic representation of ToThyCholRu lodged into a bilayer. C. $\mathrm{IC}_{50}$ values of $\mathrm{AziRu}$ and ToThyCholRu/POPC liposome in the indicated cell lines following 72 hours of incubation. Reproduced with permission. ${ }^{[9]}$ Copyright 2012, American Chemical Society. 
A

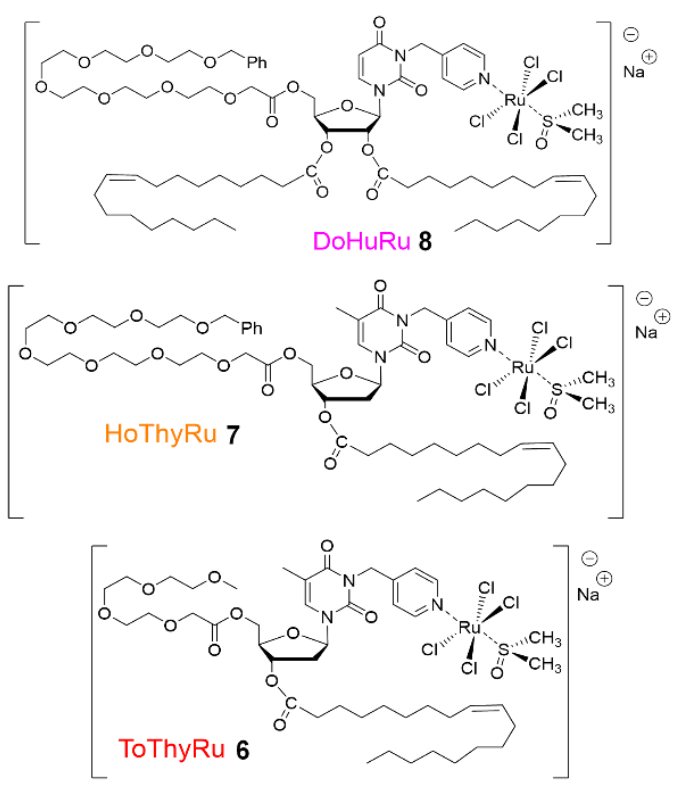

B

Hydrodynamic radius distributions

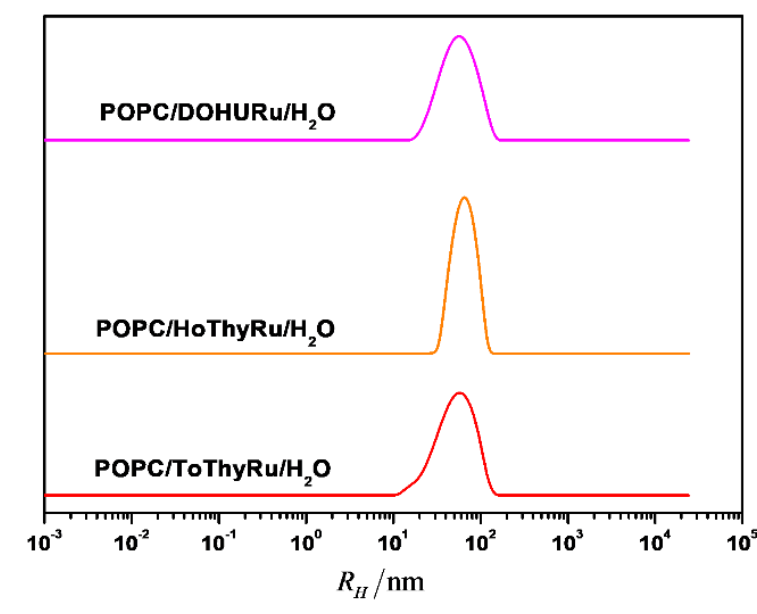

Figure 4. Ruthenium-based complex nanocarriers for cancer therapy. A. Chemical structures of ToThyRu 6 ,

HoThyRu 7 and DoHuRu 8. B. Hydrodynamic radius distributions in water for ternary systems $\mathrm{POPC} /[\mathrm{Ru}$ complex $] / \mathrm{H}_{2} \mathrm{O}$ at $85 / 15$ molar ratio between the phospholipid and $0.1 \mathrm{mmol} / \mathrm{kg}$ solution of the ruthenium complex. Reproduced with permission. ${ }^{[11]}$ Copyright 2012, Elsevier.

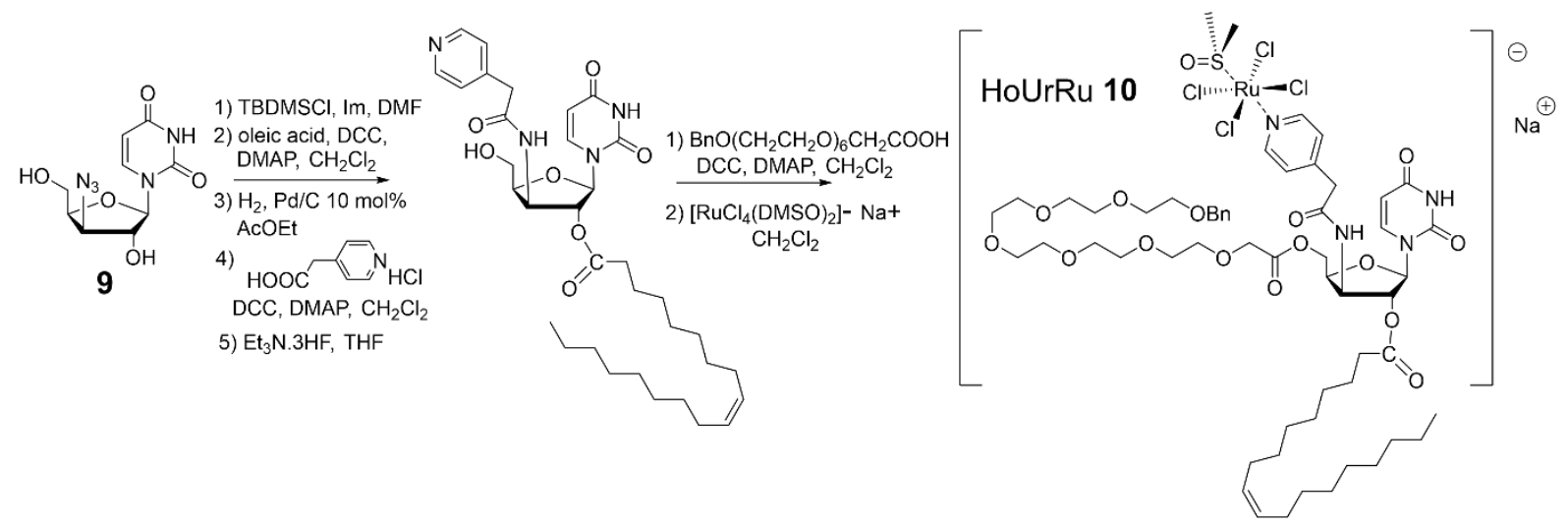

Figure 5. HoUrRu complex 10 synthesis. ${ }^{[12]}$ 
A

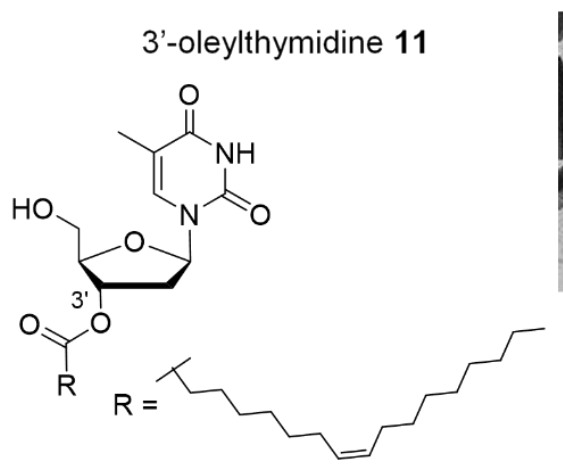

3'-phytanoylthymidine 12

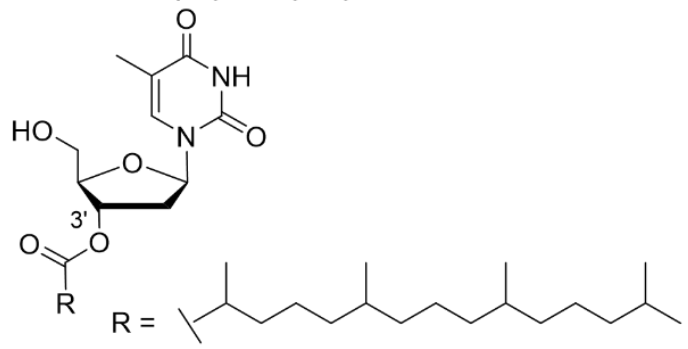

B

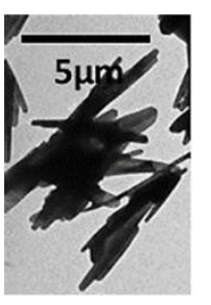

C
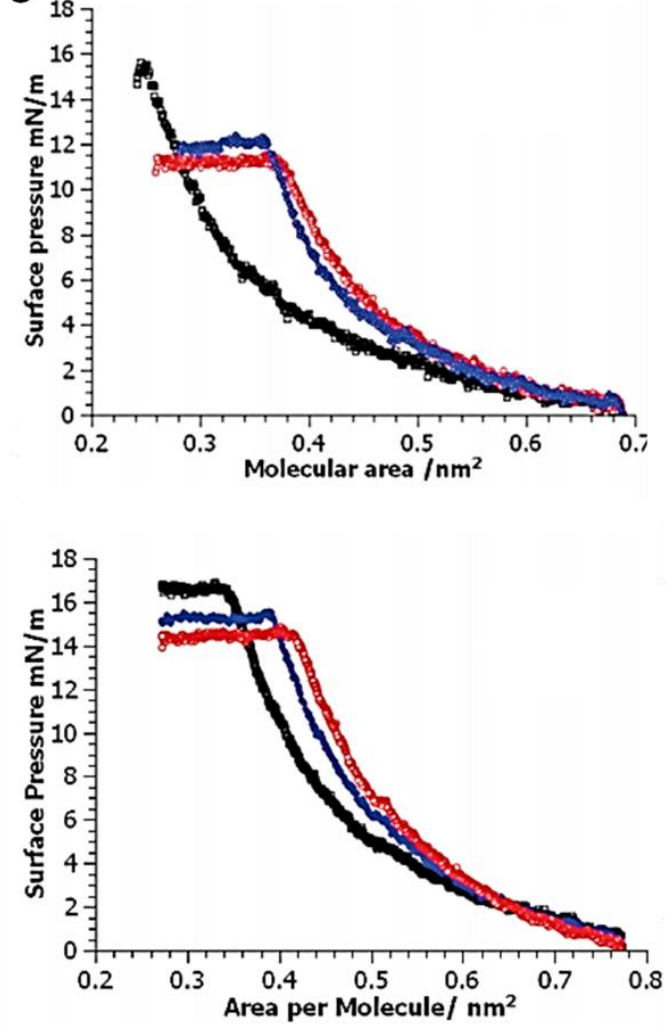

Figure 6. Nonionic thymidine nucleolipids 11, 12. A. NL chemical structures. B. TEM image of spherulites from 3'-oleoylthymidine 11 (scale bar: $5 \mu \mathrm{m}$ ). C. Surface pressure-area isotherms for monolayers of 3'oleylthymidine $\mathbf{1 1}$ and 3'-phytanoylthymidine 12. Blue curve: $\mathrm{H}_{2} \mathrm{O}+2.1 \mathrm{nmoles}$ of polyadenosine oligonucleotide (10-mer); red curve: $\mathrm{H}_{2} \mathrm{O}+2.1$ nmoles of polythymidine oligonucleotide (10-mer); black curve: $\mathrm{H}_{2} \mathrm{O}$. Reproduced with permission. ${ }^{[15]}$ Copyright 2010, American Chemical Society.
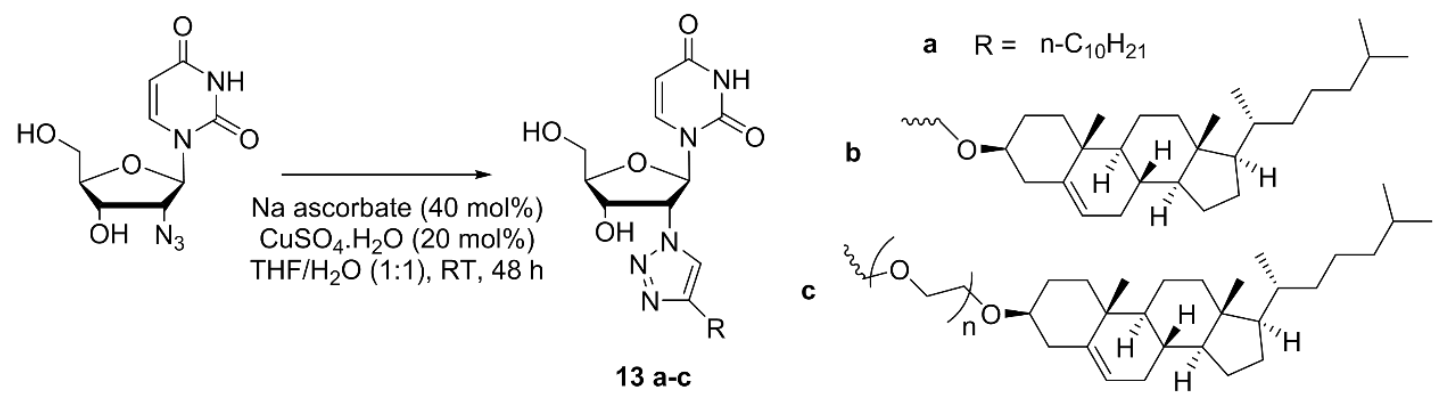

Figure 7. Synthesis approach of 2 '-substituted uridine. ${ }^{[16]}$ 


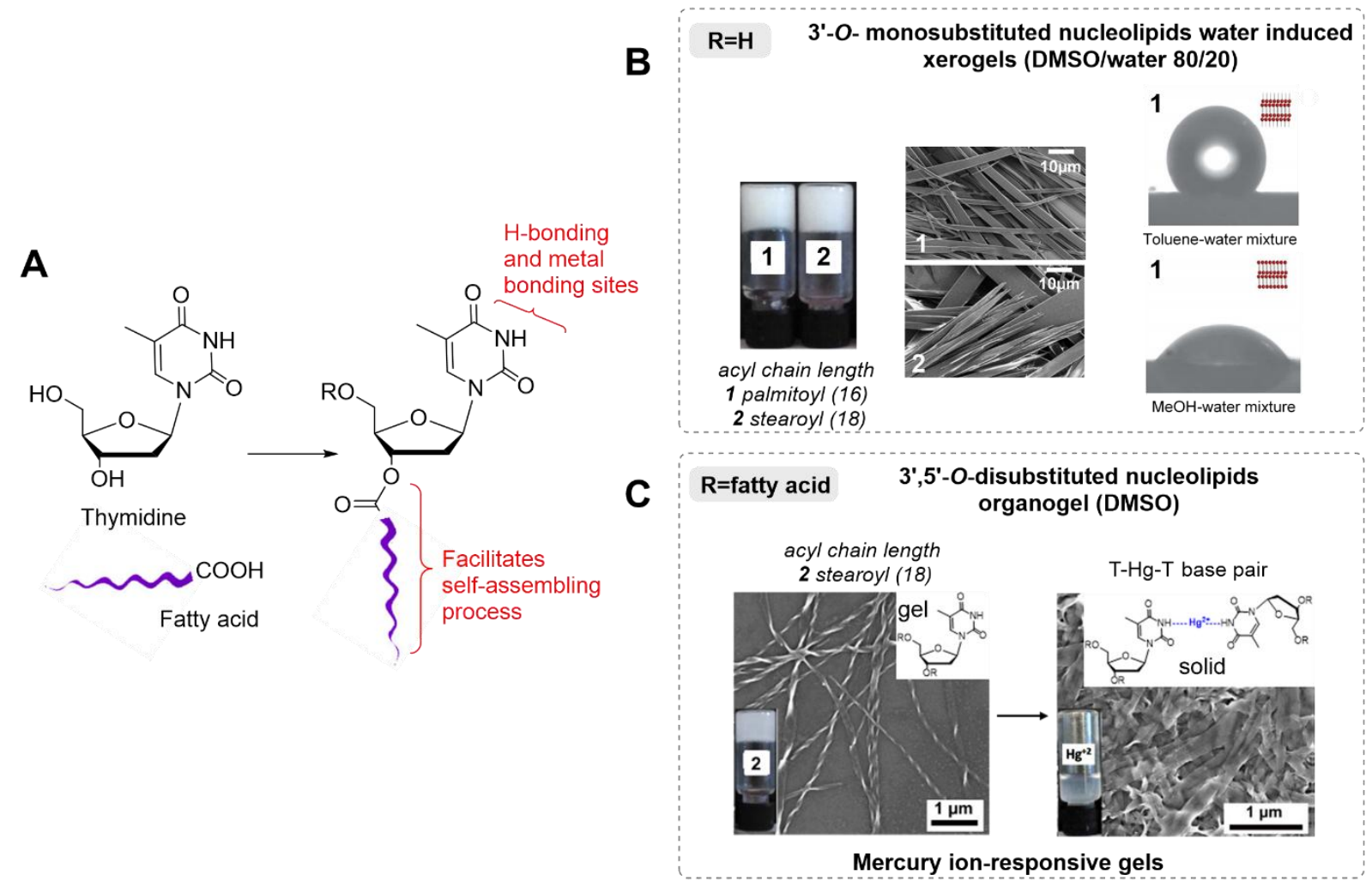

Figure 8. Supramolecular gels based on nucleolipids. A. NL chemical structures. B. 3'-O-monosubstituted xerogels 1 and 2 gelation tests (DMSO/water 80/20), FESEM images of 1 (top; scale bar: $10 \mu \mathrm{m}$ ) and 2 (bottom; scale bar: $10 \mu \mathrm{m}$ ) and contact angle images of water droplets on the surface of 1 fabricated from toluene-water mixture (top) and methanol-water mixture (bottom). C. 3', 5'-O-disubstituted 2 gelation tests and FESEM images without (left) and with mercury ions (right). Reproduced with permission. ${ }^{[17]}$ Copyright 2017, American Chemical Society. 


\section{0-2',3'-ketal 5-fluorouridine derivatives}

A
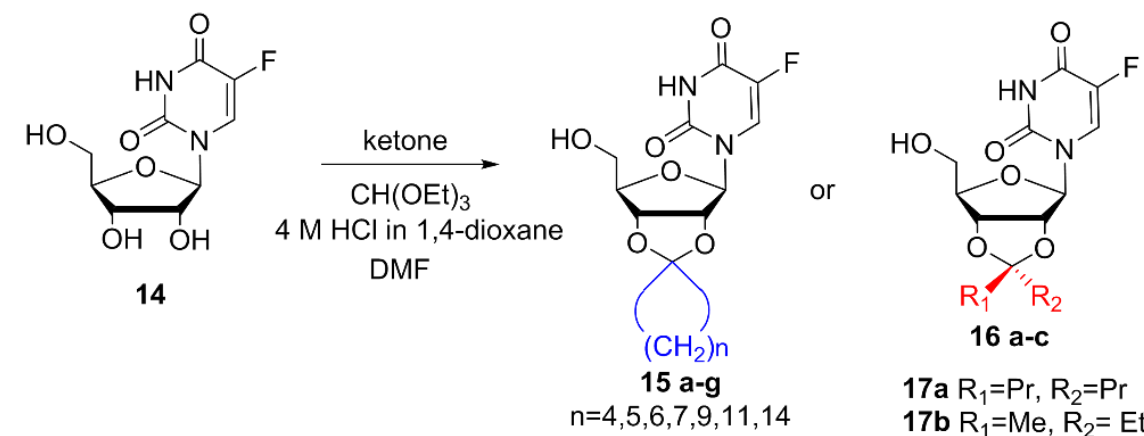

17a $\mathrm{R}_{1}=\mathrm{Pr}, \mathrm{R}_{2}=\mathrm{Pr}$

17b $\mathrm{R}_{1}=\mathrm{Me}, \mathrm{R}_{2}=\mathrm{EtOOCCH} \mathrm{CH}_{2}$

17c $\mathrm{R}_{1}=\mathrm{Me}, \mathrm{R}_{2}=4-\mathrm{MeC}_{6} \mathrm{H}_{4} \mathrm{COO}\left(\mathrm{CH}_{2}\right)_{3}$

\section{Nucleoterpenes}

B

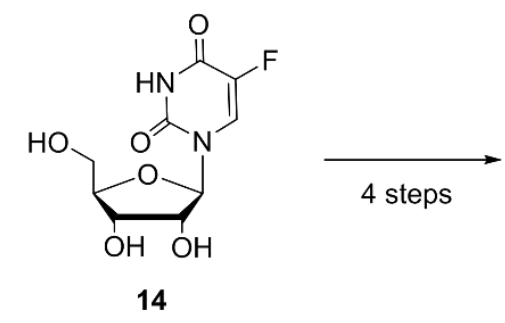<smiles></smiles>

a: $\mathrm{R}=$<smiles>CCCC=C(C)CCCC(C)CCCC(C)CCCC(C)C</smiles>

$\mathrm{b}: \mathrm{R}=$

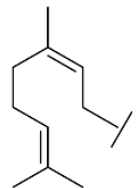

Figure 9. 5-fluorouridine-based nucleoside lipids. A. Synthetic pathway of $O-2^{\prime}, 3^{\prime}$-ketal 5-fluorouridine derivatives. ${ }^{[18]} \mathbf{B}$. Synthetic procedure of nucleoterpenes. ${ }^{[21]}$

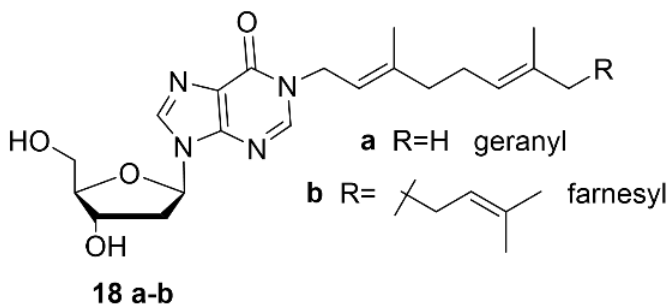

2'-deoxyinosine nucleoterpene

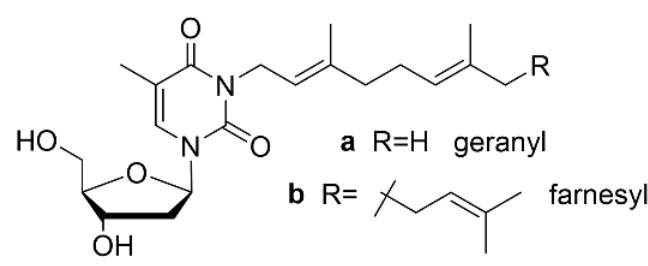

19 a-b

Thymidine nucleoterpene

Figure 10. Molecular structures of 2'-deoxyinosine and thymidine nucleoterpenes. ${ }^{[22]}$ 

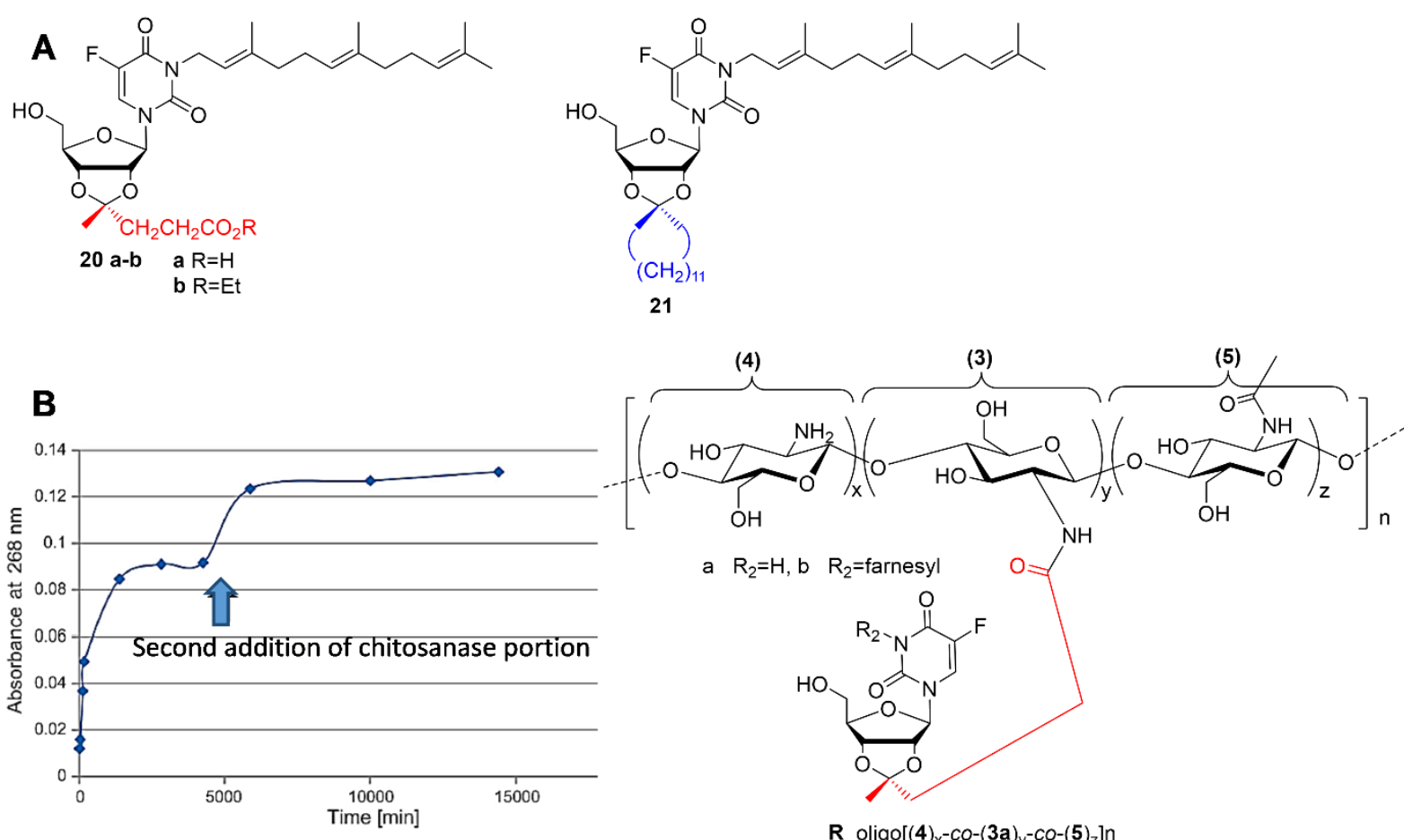

$$
\begin{aligned}
& \mathbf{R} \text { oligo }\left[(4)_{x}-c o-(3 a)_{y}-c o-(5)_{z}\right] n \\
& \mathbf{S} \text { oligo }\left[(4)_{x}-c o-(3 b)_{y}-c o-(5)_{z}\right] n
\end{aligned}
$$

Figure 11. Covalent immobilization of farnesylated nucleolipids on chitosans. A. NL chemical structures. B. Chitosanase-catalyzed degradation of a 5-fluorouridine chitosan-foil conjugate as a function of time. Reproduced with permission. ${ }^{[23]}$ Copyright 2013, Wiley-VCH.
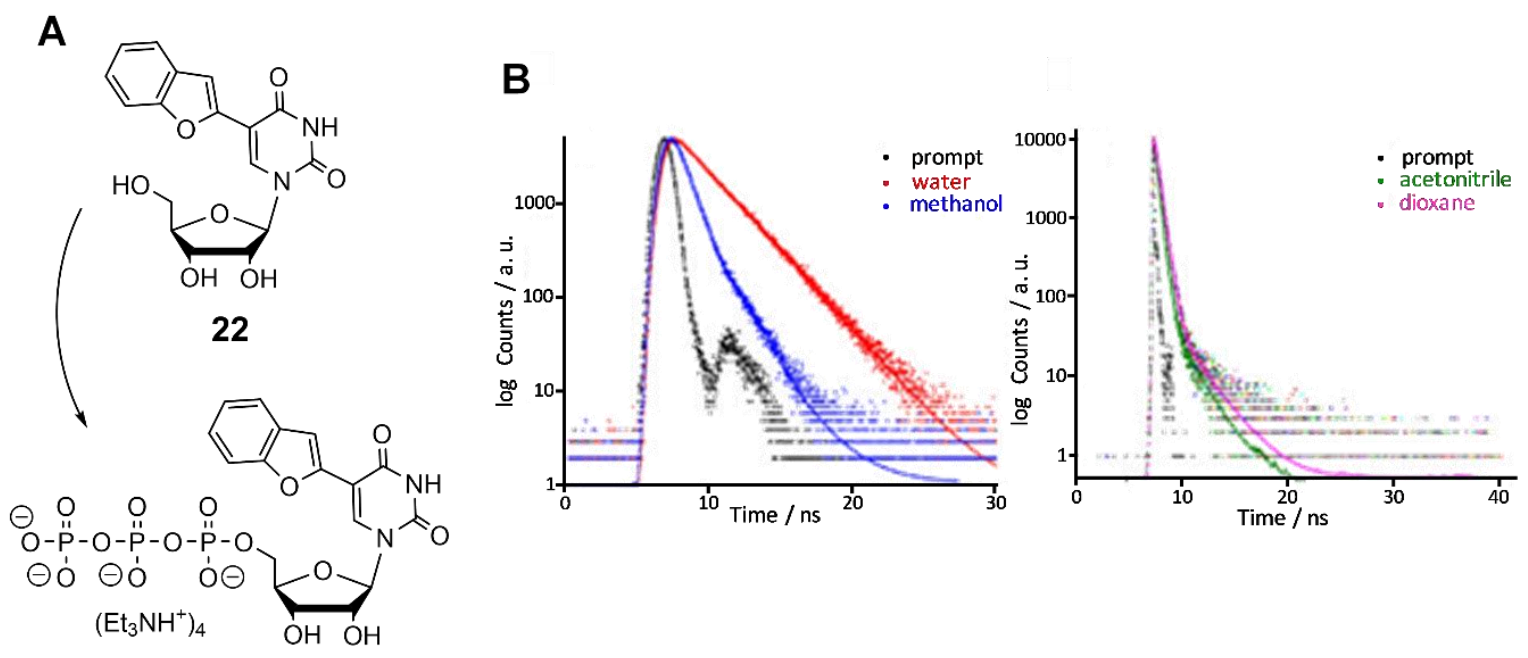

23

Figure 12. Fluorescent pyrimidine ribonucleoside analog. A. Chemical structures of probes $\mathbf{2 2}$ and 23. B. Excited state decay profile of ribonucleoside $\mathbf{5}$ in water (red), methanol (blue), acetonitrile (green) and dioxane (pink). Laser profile in black (prompt). Reproduced with permission. ${ }^{[26]}$ Copyright 2011, Wiley-VCH. 


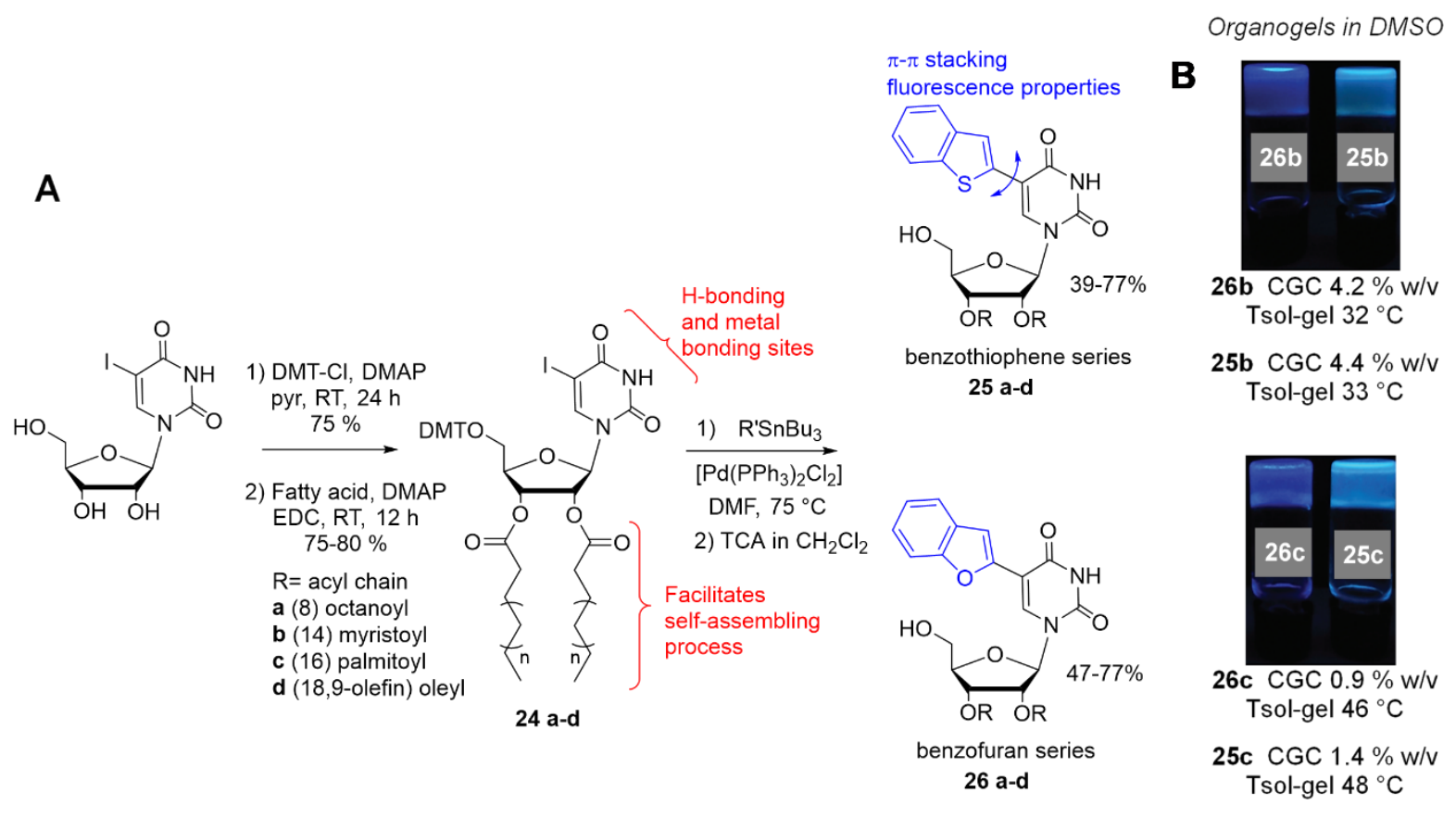

Figure 13. Responsive fluorescent nucleolipid gels. A. NLs $\mathbf{2 5}$ and $\mathbf{2 6}$ synthetic pathway. B. Gel images under UV light (top, $365 \mathrm{~nm}$ ) and gelation properties of fluorescent NLs (bottom). Reproduced with permission. ${ }^{[28]}$ Copyright 2016, Royal Society of Chemistry. 
A

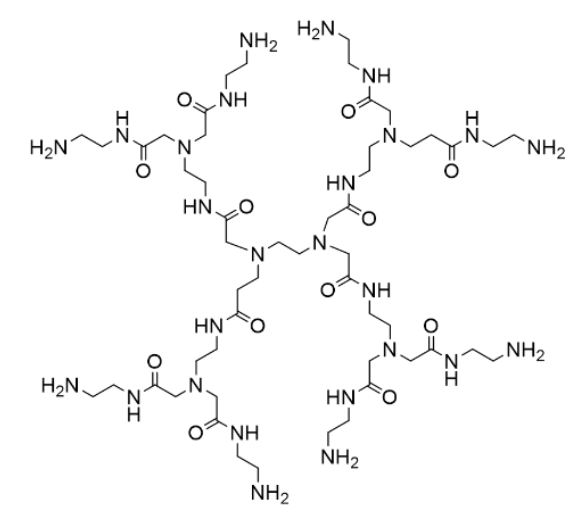

Generation 1

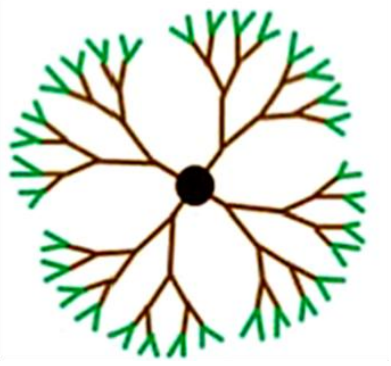

Generation 4

poly(amidoamine) (PAMAM) dendrimer
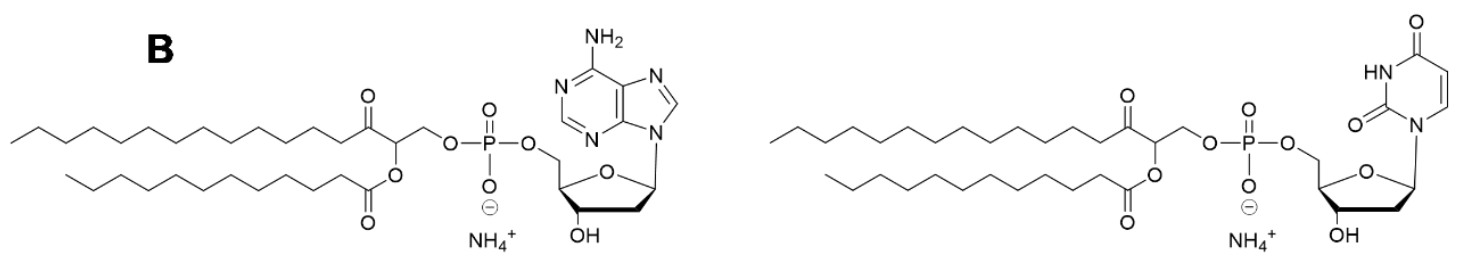

1,2-dilauroyl-sn-glycero-3-phosphoadenosine (DLPA) 1,2-dilauroyl-sn-glycero-3-phosphouridine (DLPU)

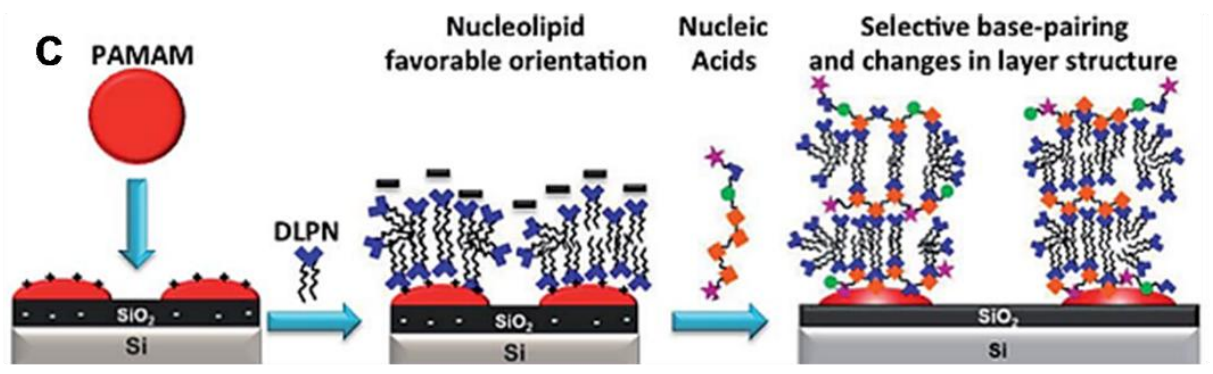

Figure 14. Dendrimer/nucleotide lipid surface films. A. PAMAM dendrimers. B. Nucleotide lipids (DLPNs),

1,2-dilauroyl-sn-glycero-3-phosphoadenosine (DLPA) and 1,2-dilauroyl-sn-glycero-3-phosphouridine (DLPU).

C. Sequential addition of dendrimers, DLPN and nucleic acids allowing selective binding between the functionalized surface and nucleic acids. Reproduced under the terms of CC-BY-3.0 license. ${ }^{[34]}$ Copyright 2015, Royal Society of Chemistry. 
B 27a nucleolipid film

A

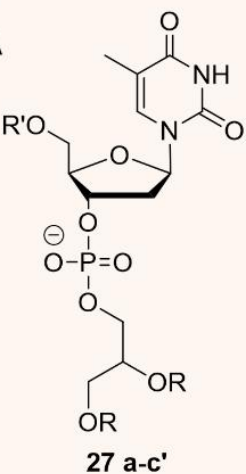

a $\mathrm{R}=$ palmitoyl $(16) \mathrm{R}^{\prime}=\mathrm{H}$

b R= myristoyl (14) R'= H

c $\mathrm{R}=$ oleyl $(18: 1) \mathrm{R}^{\prime}=\mathrm{H}$

b' $\mathrm{R}=$ myristoyl (14) $\mathrm{R}^{\prime}=\mathrm{CH}_{2} \mathrm{Ph}$ c' R= oleyl (18:1) R'= $\mathrm{CH}_{2} \mathrm{Ph}$

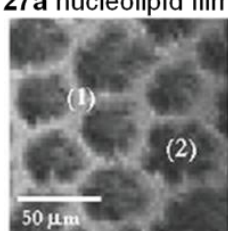

C 27 b-c unilamellar vesicles 27 b'-c' multilamellar vesicles
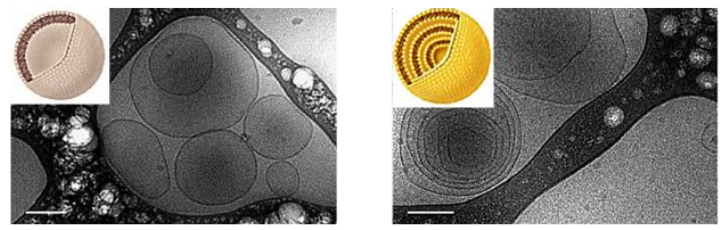

D 27a hydrogels

(4.) Self assembly
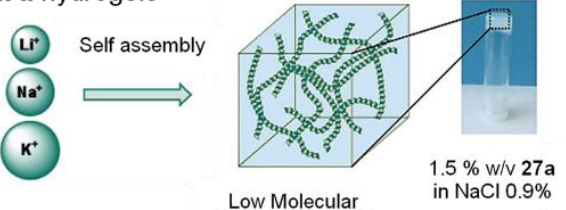

Low Molec in $\mathrm{NaCl} 0.9 \%$

Figure 15. Colloidal properties of 1,2-diacyl-sn-glycerol nucleotide lipids. A. Chemical structures of nucleotide lipids. B. Ellipsometric image for a film of 27a under compression representing (1) monolayer and (2) bilayer (scale bar: $50 \mu \mathrm{m}$ ). Reproduced with permission. ${ }^{[42]}$ Copyright 2012, American Chemical Society. C. Example of CryoTEM images of unilamellar (top) and multilamellar (bottom) vesicular systems formed by 27b and 27b' respectively (scale bar: $200 \mathrm{~nm}$, HEPES buffer $50 \mathrm{mM}$ ). Reproduced with permission. ${ }^{[43]}$ Copyright 2013, American Chemical Society. D. $1.5 \%$ w/v low molecular weight hydrogel of $\mathbf{2 7 a}$ formed in $0.9 \% \mathrm{NaCl}$ in water. Reproduced with permission. ${ }^{[44]}$ Copyright 2017, Wiley-VCH. 


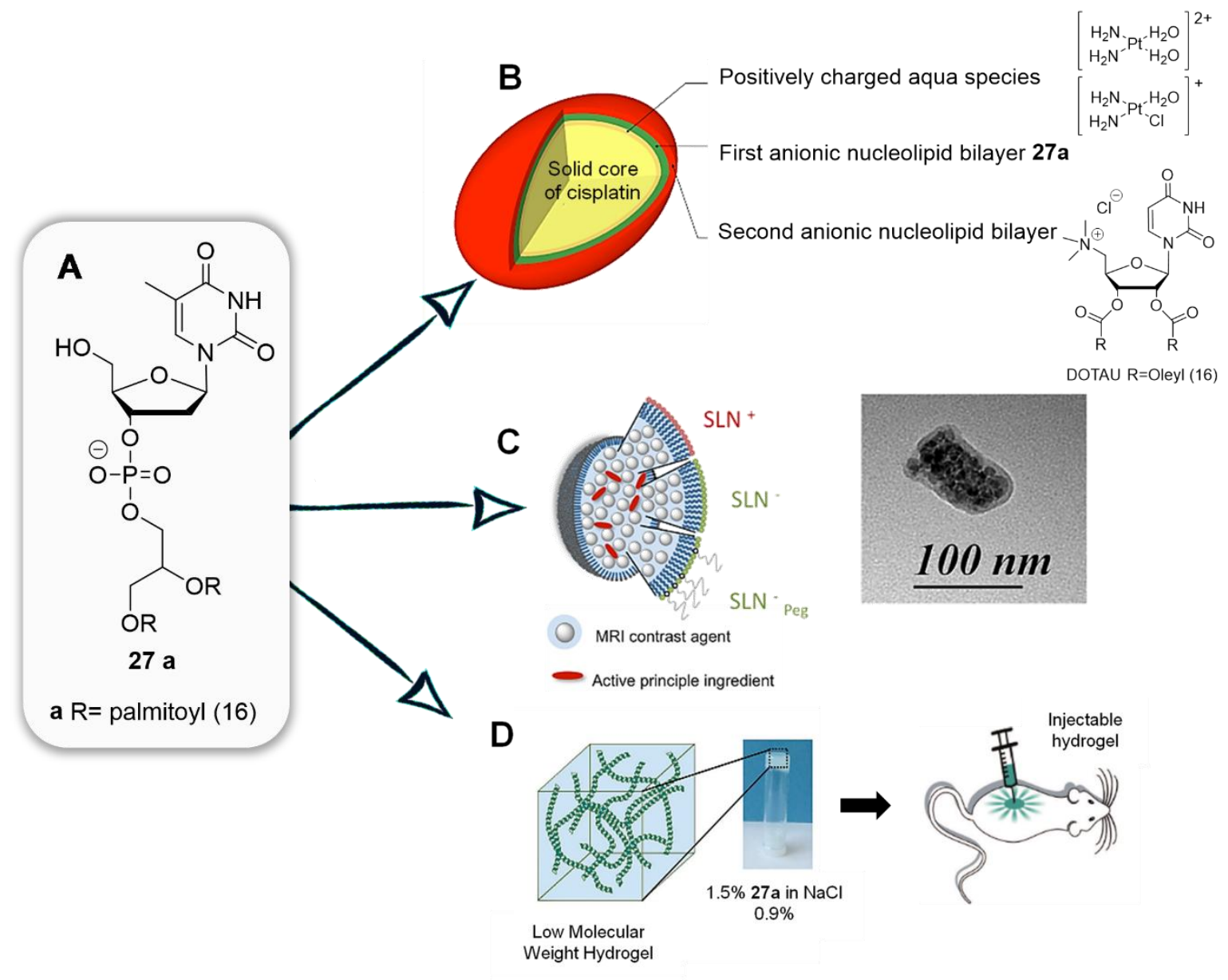

Figure 16. Examples of nucleotide lipid biomedical applications. A. Chemical structure of 27a. B. Nanoparticle schematic representation including anionic (27a) and cationic (DOTAU, 2', 3'-dioleyl-5' -deoxy-5'trimethylammoniumuridine) NLs. Reproduced with permission. ${ }^{[47]}$ Copyright 2011, American Chemical Society. C. Nucleotide lipid-based SLN schematic representation (left) and TEM image of iron nanoparticles incorporated in this system (right, scale bar: $100 \mathrm{~nm}$ ). Reproduced with permission. ${ }^{[48]}$ Copyright 2016, American Chemical Society. D. Injectable hydrogel allowing drug release. Reproduced with permission. ${ }^{[44]}$ Copyright 2017, Wiley-VCH. 
A

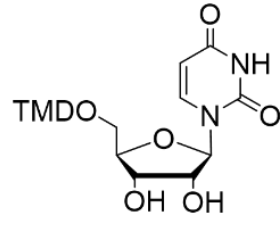

1) $C D I$ (2.2 eq), DMAP (cat. amount) Anh DMF, RT, $1 \mathrm{~h}$

Oleylamine (7 eq), RT, $12 \mathrm{~h}$

2) $\mathrm{Cl}_{3} \mathrm{CCOOH}$ in $\mathrm{DCM}$ (excess) $\mathrm{RT}, 30 \mathrm{~min}$

3)

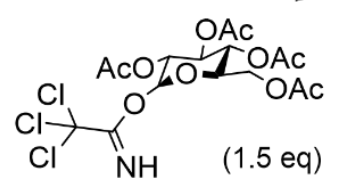

$\mathrm{BF}_{3} . \mathrm{Et}_{2} \mathrm{O}(1.5 \mathrm{eq})$, Molecular sieves DCM, RT, $4 \mathrm{~h}$

4) $\mathrm{MeONa} 1 \mathrm{M}$ in $\mathrm{MeOH}$, Dry $\mathrm{MeOH}$ $\mathrm{RT}, 4 \mathrm{~h}$

B

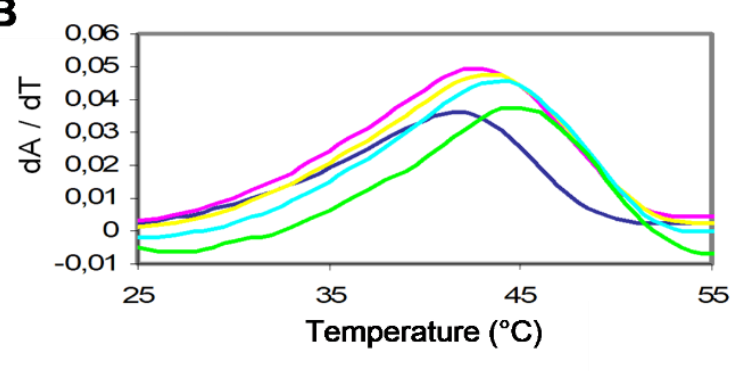

C

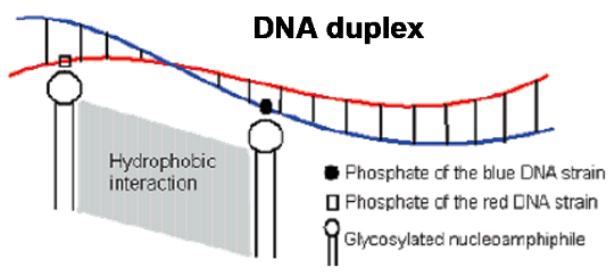

$\begin{array}{rrl}\text { - PolyA-PolyU PolyA-PolyU, [compound]/[phosphate] } & =1 \\ 4 & \\ 8 & 10=\end{array}$

Figure 17. First designed GNL as an interesting tool for DNA stabilization. A. Glycosyl dicarbamate uridine synthesis. B. Absorbance derivative at $260 \mathrm{~nm}$ depending on temperature of polyA-polyU in presence of increasing amounts of GNL. C. DNA duplex stabilization by hydrophobic interactions between lipid chains and hydrogen bonding between sugars and phosphates. Reproduced with permission. ${ }^{[50]}$ Copyright 2005, American Chemical Society. 
A

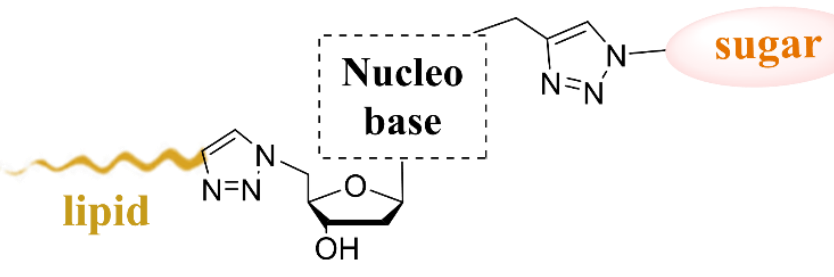

GNL

B

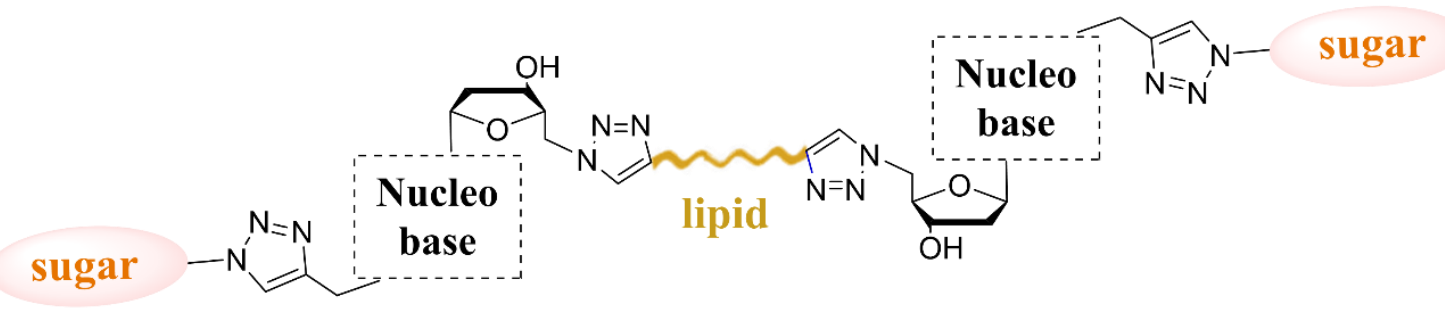

GNBA

Figure 18. Schematic representation of A. GNL and B. GNBA.
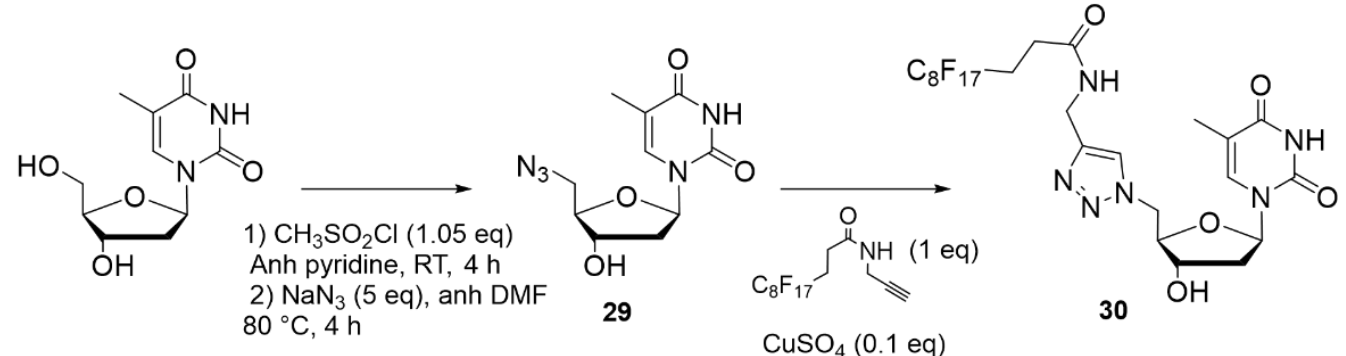

$\mathrm{CuSO}_{4}(0.1 \mathrm{eq})$

$\mathrm{Na}$ ascorbate $(0.2 \mathrm{eq})$

$\mathrm{THF} / \mathrm{H}_{2} \mathrm{O}(1: 1)$

$65^{\circ} \mathrm{C}, 10 \mathrm{~h}$

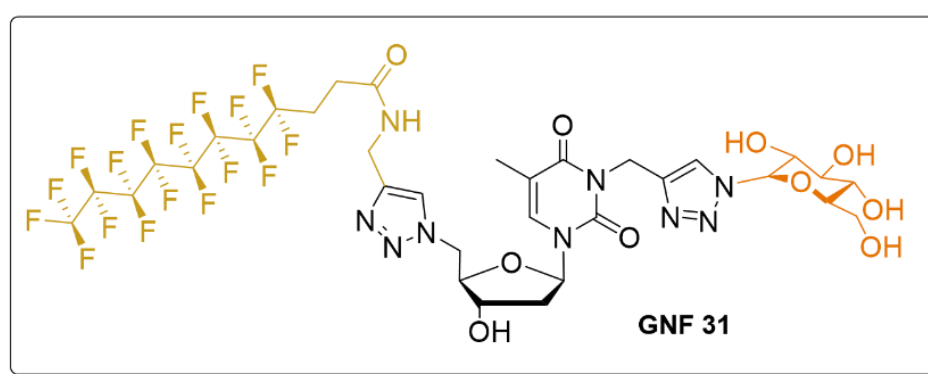

30

1) $\mathrm{HCCCH}_{2} \mathrm{Br}(2 \mathrm{eq})$

$\mathrm{K}_{2} \mathrm{CO}_{3}(2 \mathrm{eq})$

Anh DMF, RT, overnight

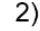

$\mathrm{CusO}_{4}(0.1 \mathrm{eq})$
$\mathrm{Na}$ ascorbate $(0.2 \mathrm{eq})$
$\mathrm{THF} / \mathrm{H}_{2} \mathrm{O}(1: 1), 65^{\circ} \mathrm{C}, 10 \mathrm{~h}$

Figure 19. Synthetic route of GNF. ${ }^{[56]}$ 


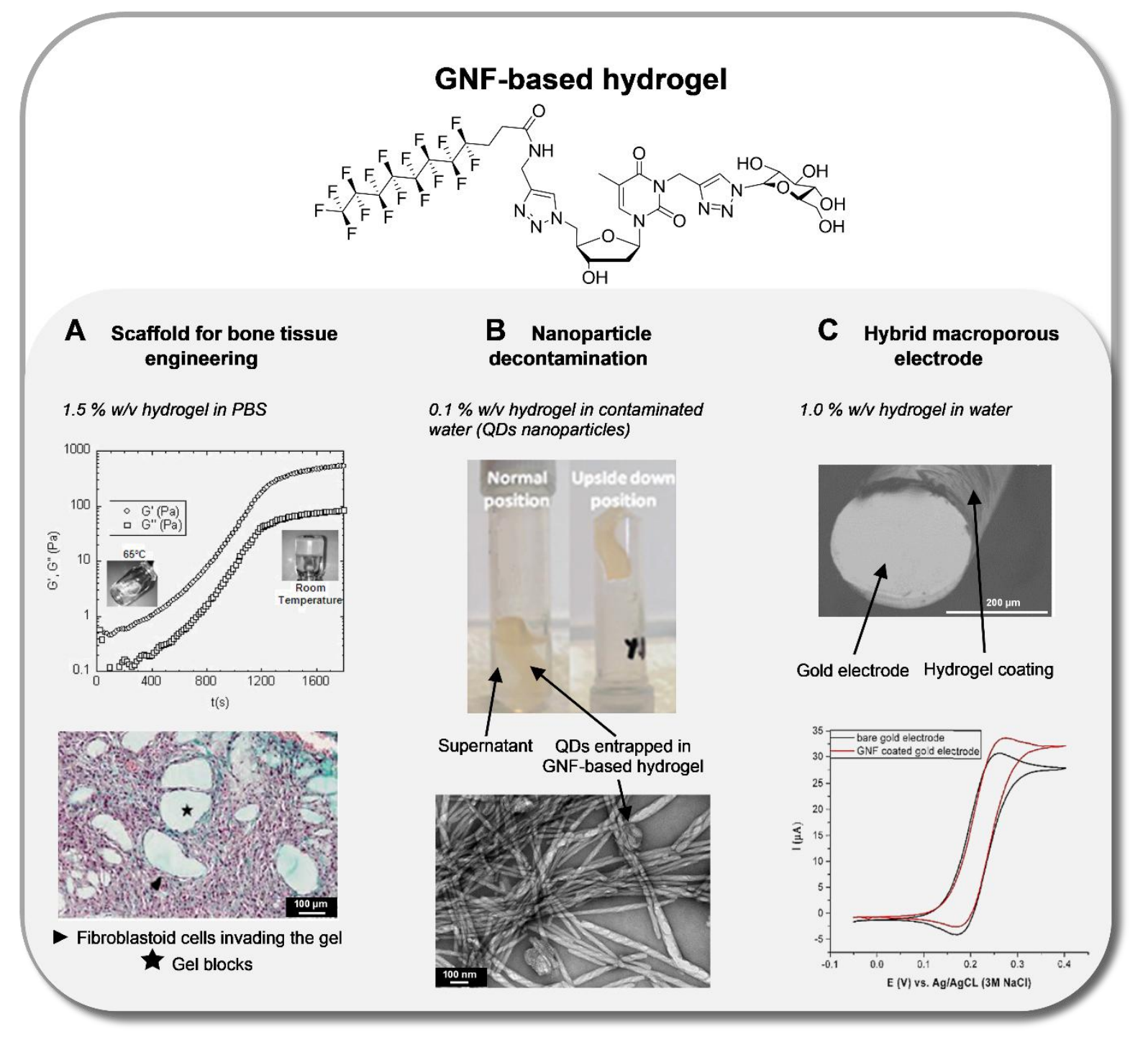

Figure 20. GNF based hydrogel - a promising tool for biomedical applications. A. Bone tissue engineering. Gelation monitoring of a $1.5 \%$ w/v GNF-based hydrogel in PBS at RT (top) and histological section of biopsies 60 days after subcutaneous implantation of GNF-based hydrogel in mice (bottom, Masson's trichrome stain, scale bar: $100 \mu \mathrm{m}){ }^{[62]}$. B. Nanoparticle decontamination. QDs entrapped in GNF-based hydrogel $0.1 \% \mathrm{w} / \mathrm{v}$ in contaminated water in normal and upside-down positions (top) and a TEM image showing entrapped QDs in the hydrogel (bottom, scale bar: $100 \mathrm{~nm}$ ). Reproduced with permission. ${ }^{[63]}$ Copyright 2015, Royal Society of Chemistry. C. Hybrid macroporous electrode. SEM image of the crossing section of the coated electrode (top, scale bar: $200 \mu \mathrm{m})$ and cyclic voltammetry of electrodes (bottom) without (black line) and with hydrogel (red line). Reproduced with permission. ${ }^{[64]}$ Copyright 2017, American Chemical Society. 
A

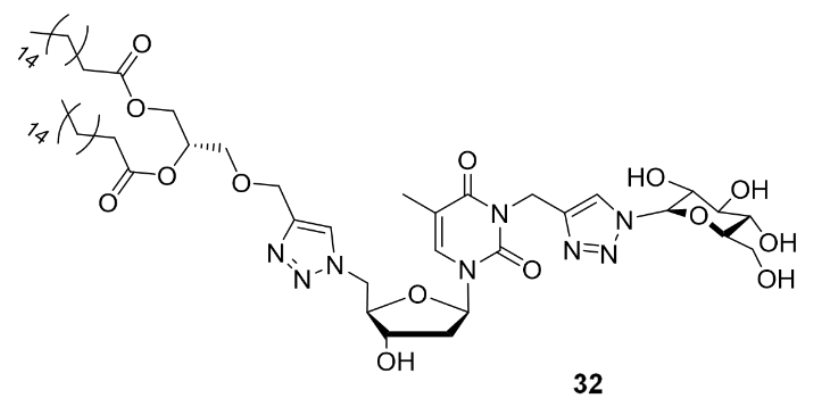

B
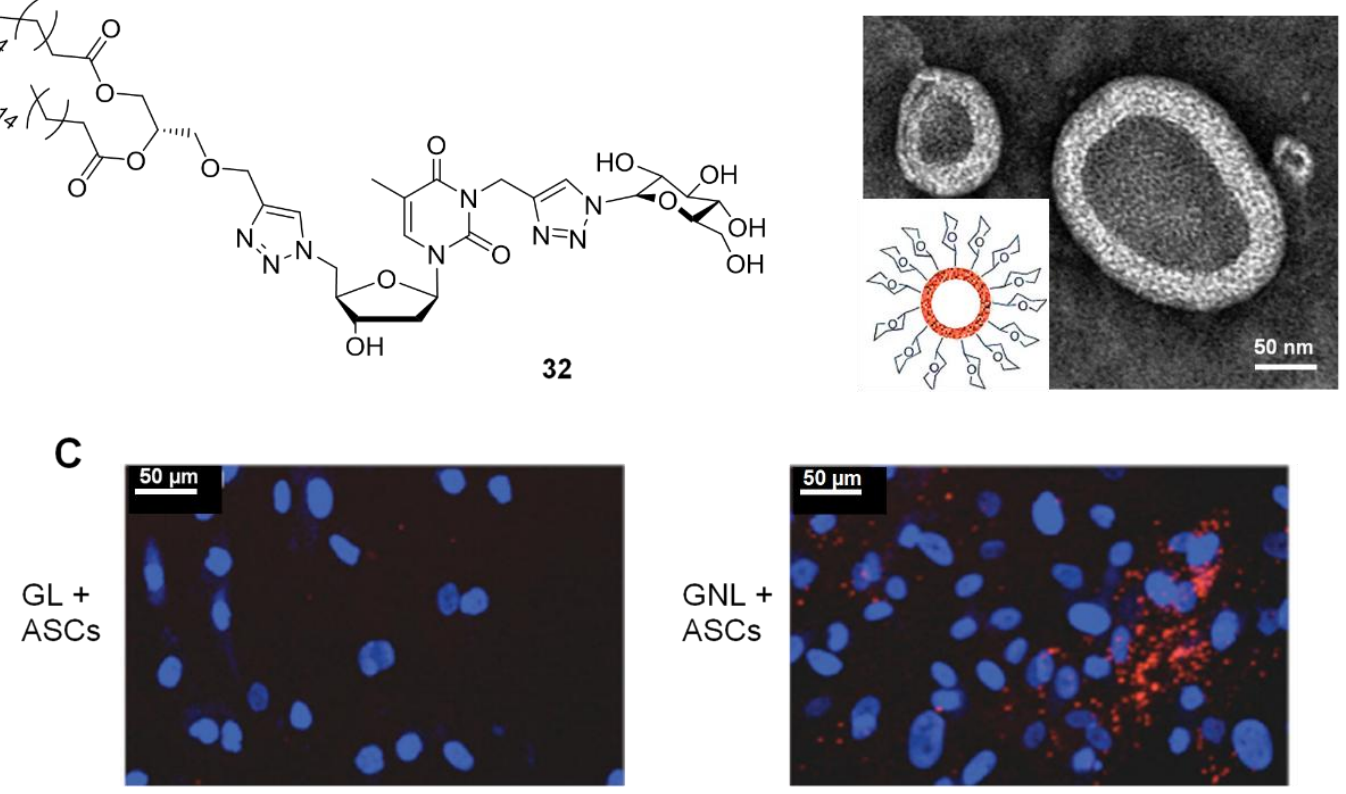

Figure 21. GNL liposome-like structure as a drug delivery system. A. GNL structure. B. TEM image of liposome-like structure (scale bar: $50 \mathrm{~nm}$ ). C. Confocal microscopy showing the internalization of DIL-labeled GL-containing liposome (top) and GNL-containing liposome (bottom) in ASCs; nuclei stained in blue (scale bar: $50 \mu \mathrm{m}$ ). Reproduced with permission. ${ }^{[57]}$ Copyright 2011, Royal Society of Chemistry.
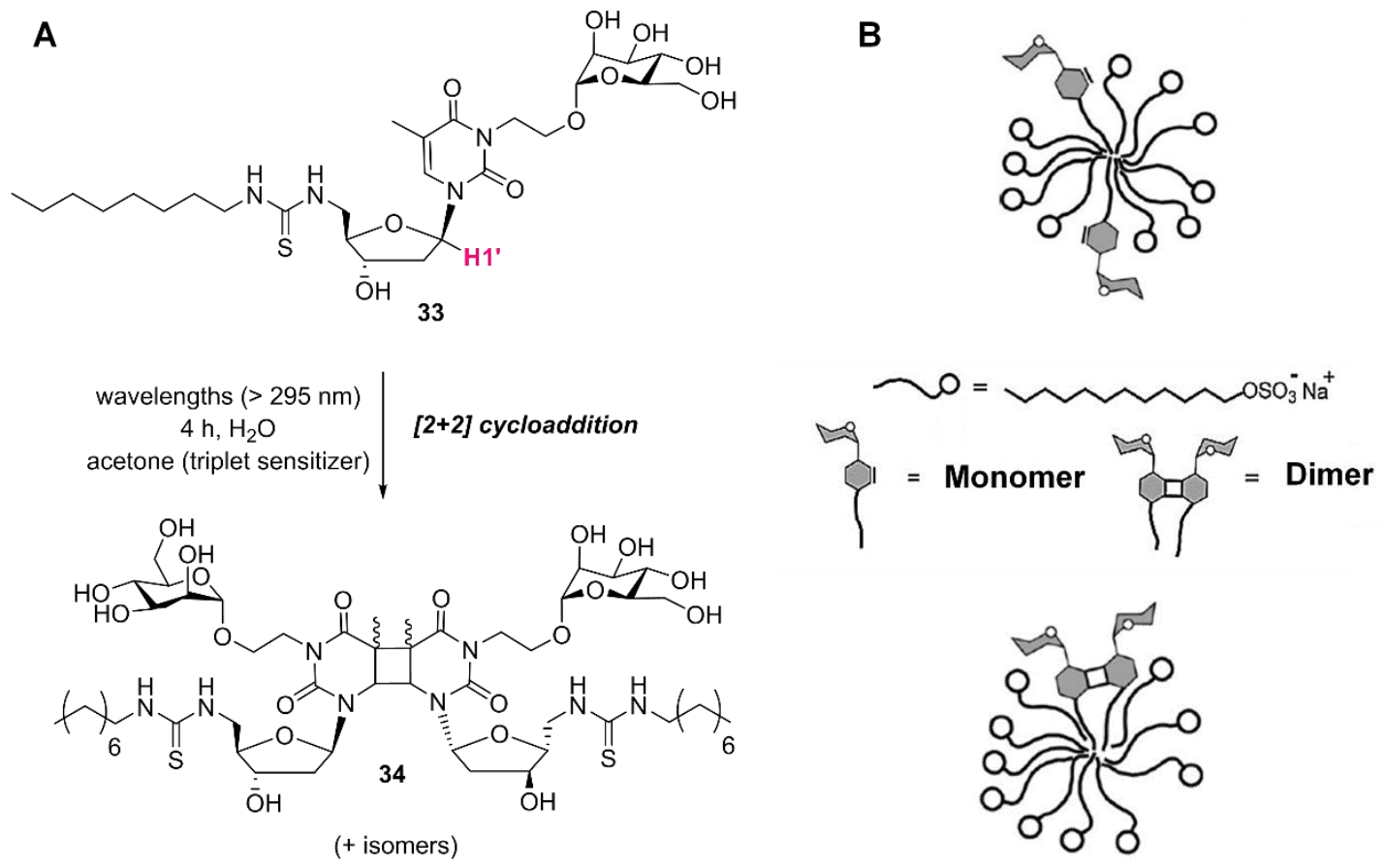
Figure 22. Photoswitchable glycothymidines. A. Molecular structures of monomers (top) and dimers (bottom). B. Schematic representation of incorporated monomers into SDS micelles and then photodimerized inside the structure. Reproduced with permission. ${ }^{[65]}$ Copyright 2011, Royal Society of Chemistry.
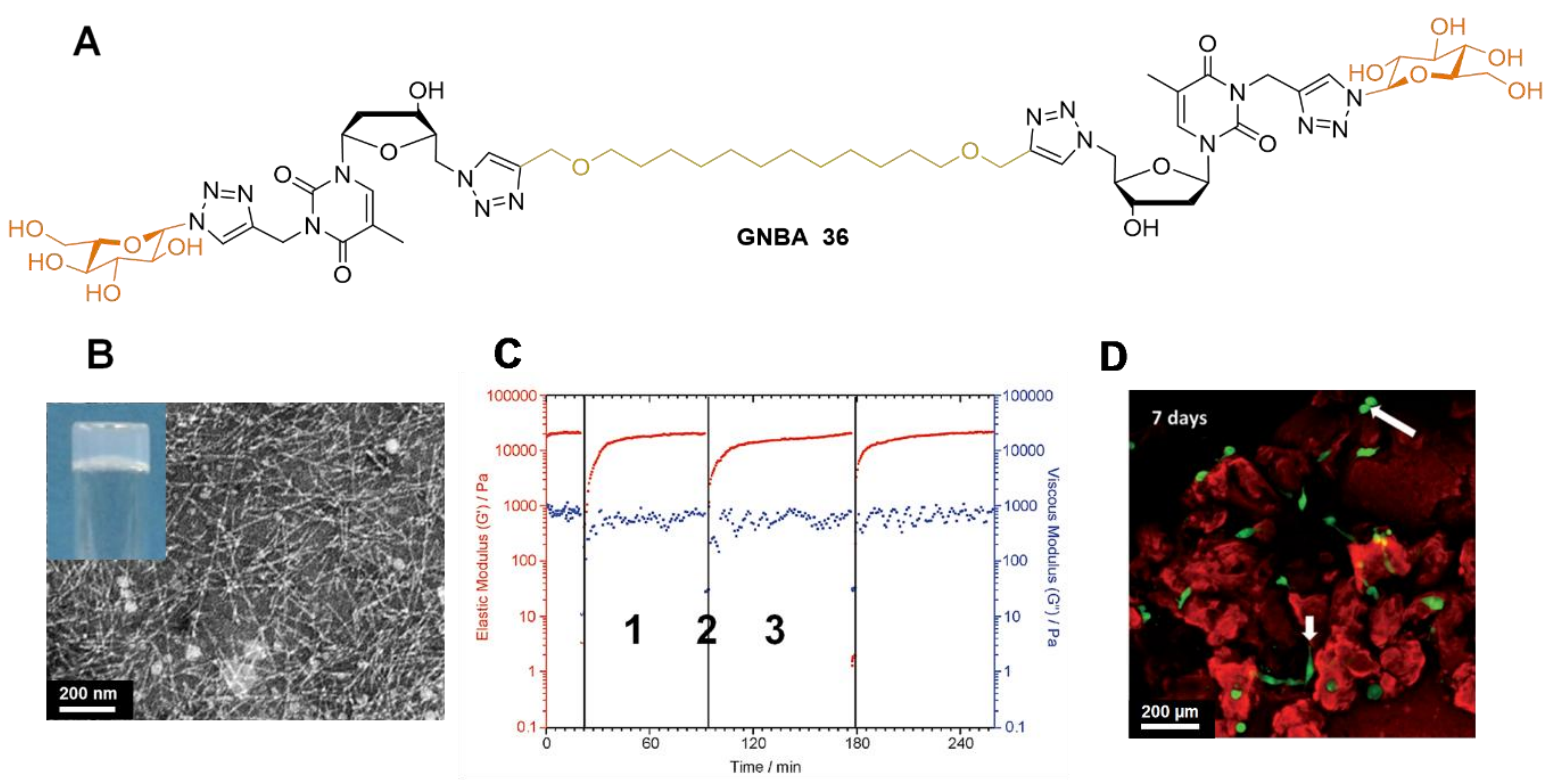

Figure 23. GNBA-based hydrogel as an extracellular matrix substitute. A. GNBA chemical structure. B. GNBA-based hydrogel $1.5 \%$ w/v TEM image (scale bar: $200 \mathrm{~nm}$ ). C. Thixotropic property of the hydrogel $\left(1 \% \mathrm{w} / \mathrm{v}\right.$ concentration) at a fixed angular frequency of $6.283 \mathrm{rad} . \mathrm{s}^{-1}(1.0 .03 \%$ strain for $20 \mathrm{~min} ; 2.15 \%$ strain for $2 \mathrm{~min}$; $3.0 .03 \%$ strain until the return of the initial gel state). D. Confocal macroscopy image of GNBAbased hydrogel ( $3 \%$ w/v concentration) embedded human ASCs after 7 days; top white arrow: dividing cell; bottom white arrow: fibroblastoid cell. Cells stained in green (calcein) and GNBA-based hydrogel in red (ethidium, scale bar: $200 \mu \mathrm{m}$ ). Reproduced with permission. ${ }^{[59]}$ Copyright 2015, Wiley-VCH. 


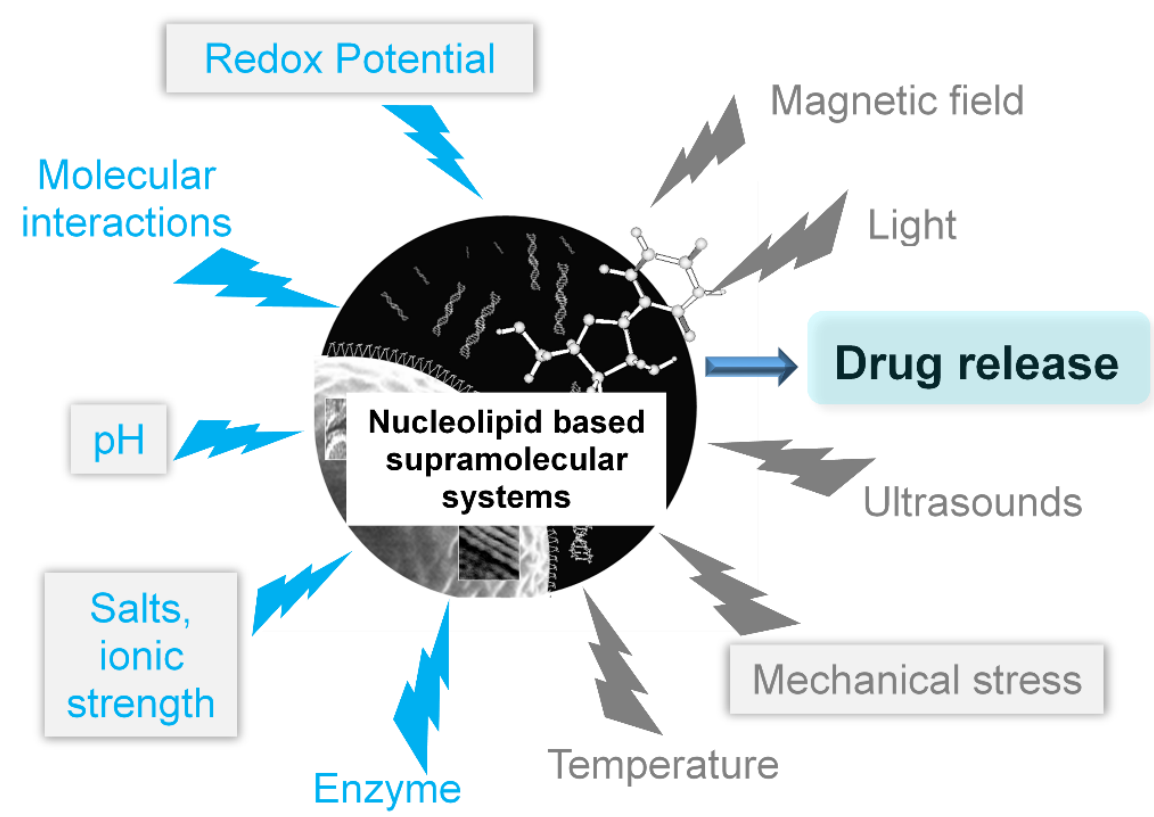

Figure 24. Schematic drawing illustrating the release of a drug from nucleolipid-based supramolecular systems upon different stimuli, either external (in gray) or internal (in blue). Gray frames indicate stimuli investigated with NLs and GNLs.

A

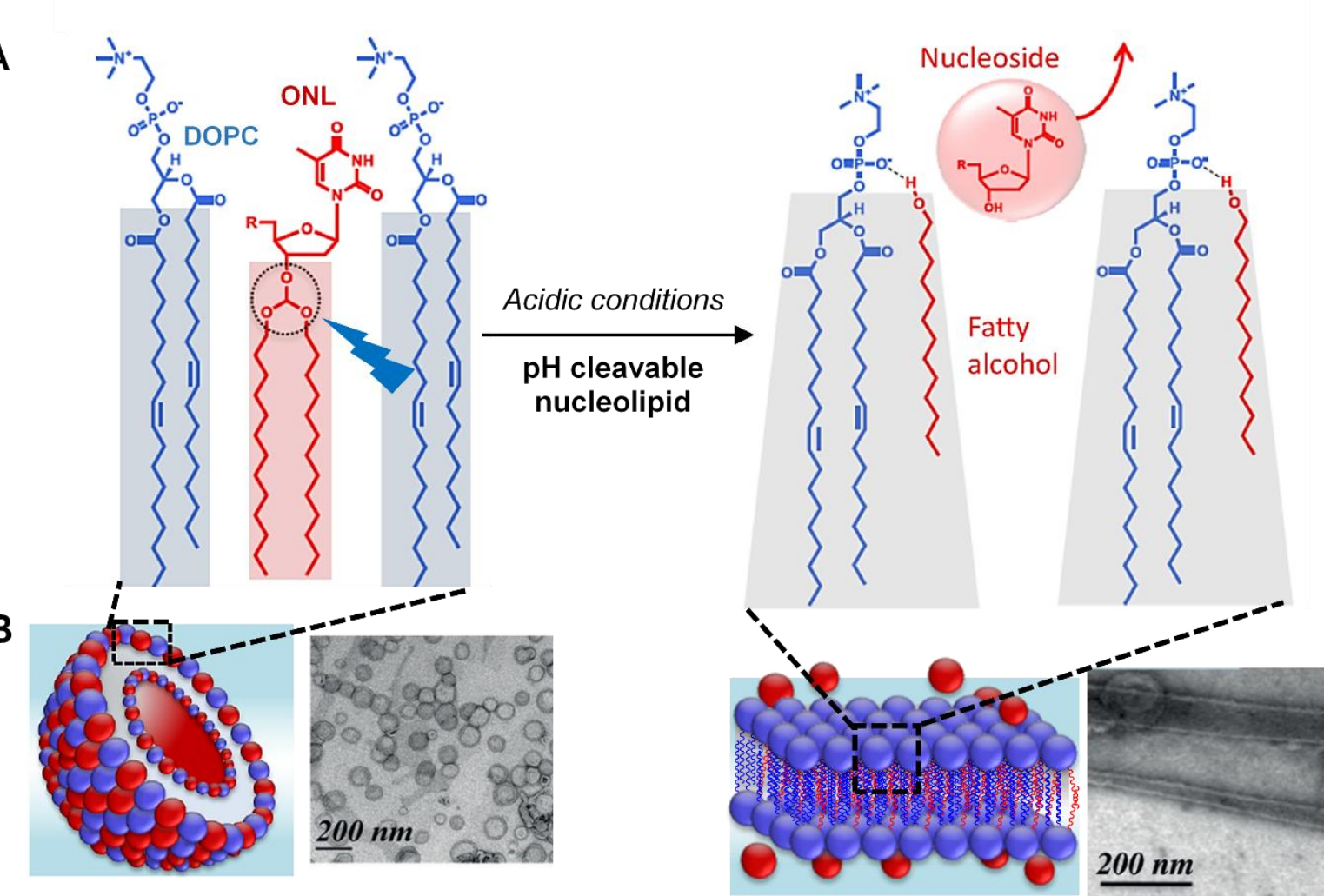

Figure 25. pH cleavable nucleolipid. A. Chemical structures of ONL/DOPC before (left) and after (right) cleavage of orthoester function (dotted line) in acidic conditions. B. Schematic representation and TEM image of 
ONL/DOPC liposomes (left, scale bar: $200 \mathrm{~nm}$ ) and their destabilization (right, scale bar: $200 \mathrm{~nm}$ ) in acidic conditions (case of anionic ONL). Reproduced with permission. ${ }^{[86]}$ Copyright 2015 , Wiley-VCH.

A

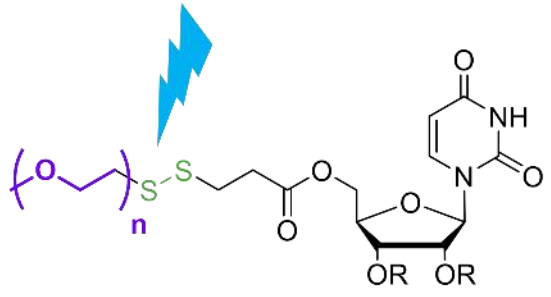

$$
R=\text { oleyl }(18: 1)
$$

DOU-SS-PEG2000

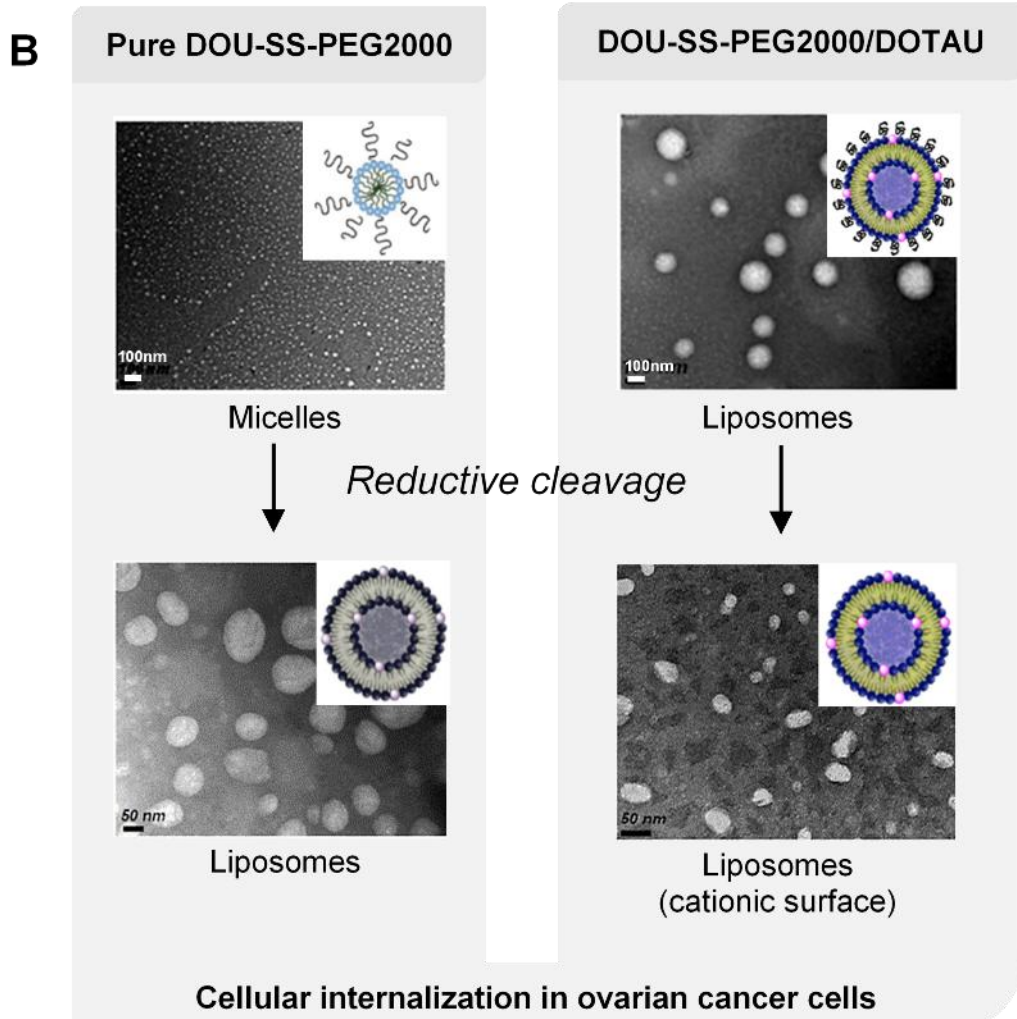

Figure 26. Cleavable PEG-based nucleolipid. A. Chemical structure of DOU-SS-PEG2000. B. TEM images of NL self-assembly to form micelles (top, scale bar: $100 \mathrm{~nm}$ ) and liposome (bottom, scale bar: $50 \mathrm{~nm}$ ) after a reductive cleavage of PEG moieties. C. TEM images of NL/DOTAU self-assembly into liposomes (top, scale bar: $100 \mathrm{~nm}$ ) and naked liposomes (bottom, scale bar: $50 \mathrm{~nm}$ ) after the reductive cleavage. Reproduced with permission. ${ }^{[89]}$ Copyright 2011, Elsevier. 


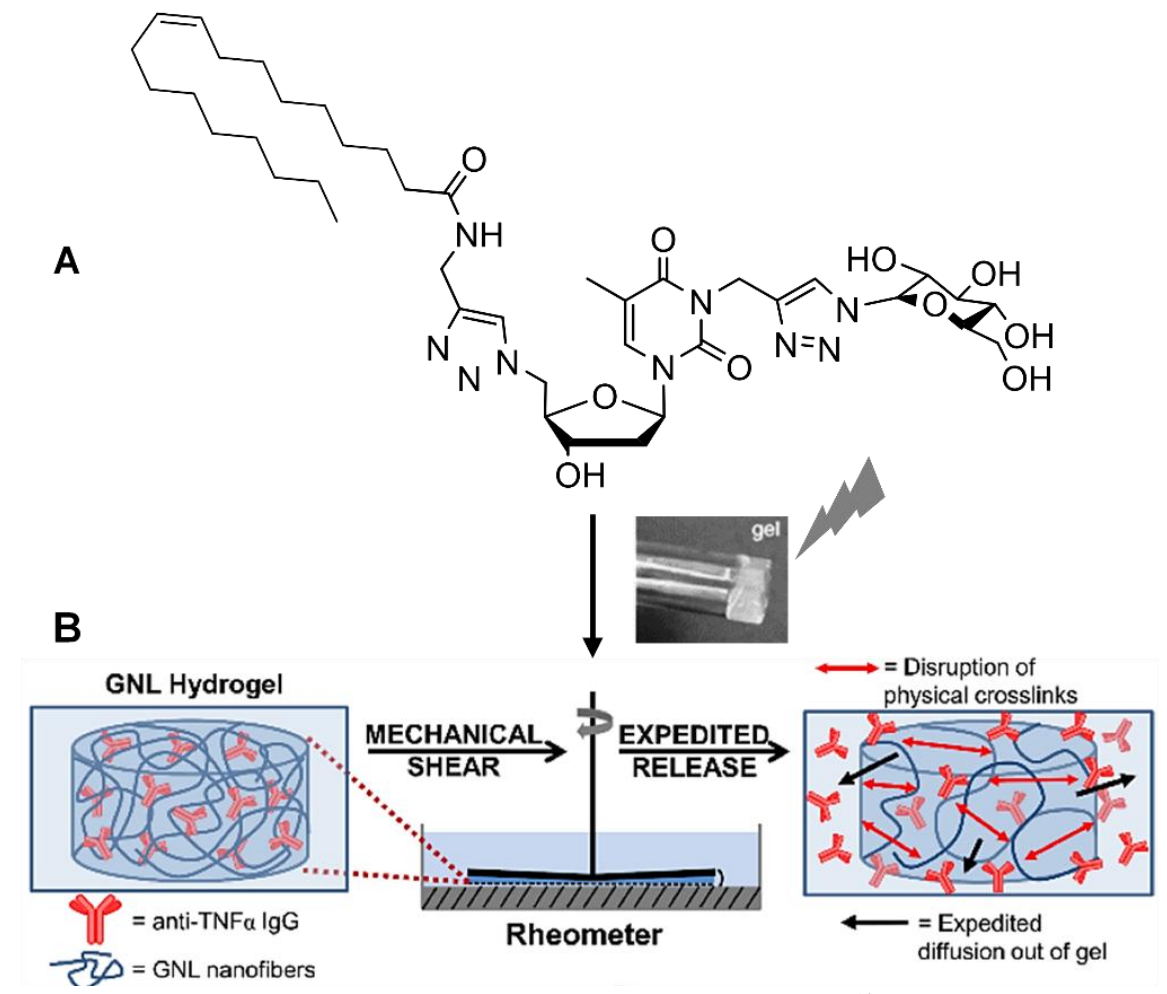

Figure 27. Mechanoresponsive hydrogel. A. GNL chemical structure. ${ }^{[54]}$ B. Schematic representation of the controlled release of anti-TNF $\alpha$ entrapped in the hydrogel upon mechanical stress. Reproduced with permission. ${ }^{[96]}$ Copyright 2016, Royal Society of Chemistry.

\section{Biographies}

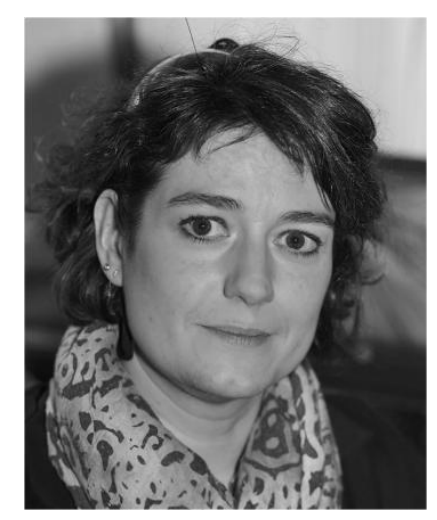

Dr. Valérie Desvergnes received her Ph.D. in chemistry from the University of Lyon 1, France in 1999. After a postdoctoral fellowship in the group of Professor Jérome Lacour (University of Geneva), she was appointed CNRS researcher at the Institute of Organic and Analytic Chemistry (ICOA, Orléans, France) in 2002. Her research topic was the design, synthesis and biological evaluation of innovative iminosugar nucleotides as antimycobacterial 
compounds. After several years at the Institute of Molecular Sciences (ISM, Bordeaux, Professor Yannick Landais' group), she joined Professor Philippe Barthélémy's team in 2016, where she develops new biomimetic organocatalyzed synthetic pathways to nucleoconjugates (nucleolipids, glyconucleolipids, etc.).

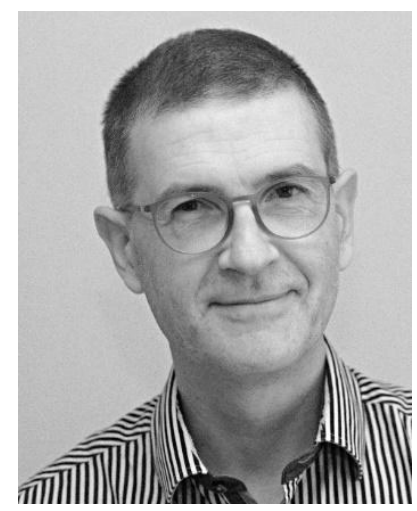

Dr. Laurent Latxague received his Ph.D. in chemistry focused on glucosinolates from the University Bordeaux I, France, in 1991, and continued his work in the group of Professor Dr. Joachim Thiem (Hamburg, Germany). He was appointed Associate Professor at the University Bordeaux 2 in 1993 in the group of Professor Gérard Déléris and was involved in the synthesis of organosilicon analogs of acyclic nucleosides. He then moved to Professor Laurence Bordenave's group (INSERM U577) in 2005 to work on biodelivery systems relating to bone regeneration. In 2011, he joined Professor Philippe Barthélémy's group to develop supramolecular hydrogels for tissue engineering.

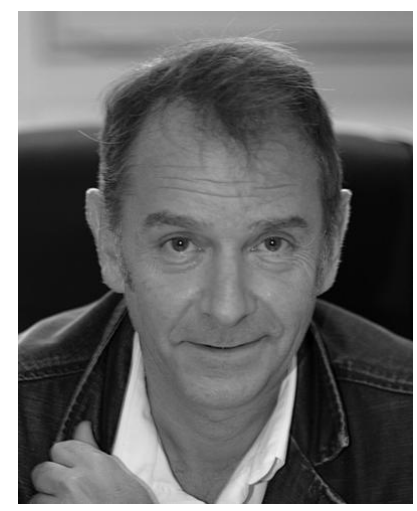


Prof. Philippe Barthélémy received his Ph.D. in chemistry from the University of Montpellier II, France in 1993. Then, he was a postdoctoral fellow at Emory University in the group of Prof. Fredric Menger (Lavoisier Grant). He was appointed Associate Professor at the University of Avignon in 1996. Barthélémy was a visiting Associate Professor at Duke University in 2001. In 2005, he was appointed full Professor at the University of Bordeaux Segalen, where he became Vice President of the University in 2011. He is leading the “ChemBioPharm" team of INSERM U1212. Over the course of his tenure, Barthélémy's research on bioinspired systems has yielded more than 130 peer-reviewed publications, 20 patents, and 110 invited conferences/presentations.

\section{Table of contents:}

Hybrid molecules combining nucleic acid structures with lipids have recently received increasing attention as a new class of supramolecular biomaterials. An overview of the latest studies on their chemical and biological properties is presented, including several biomedical applications ranging from medicinal chemistry to biomaterials. Some suggestions for developing these types of soft materials in the near future are also proposed.

Keywords: nucleosides, lipids, amphiphiles, supramolecular assemblies, hydrogels

Julie Baillet, Valérie Desvergnes, Aladin Hamoud, Laurent Latxague, and Philippe Barthélémy

Lipid and Nucleic Acid Chemistries: Combining the Best of Both Worlds to Construct Advanced Biomaterials

ToC figure: 


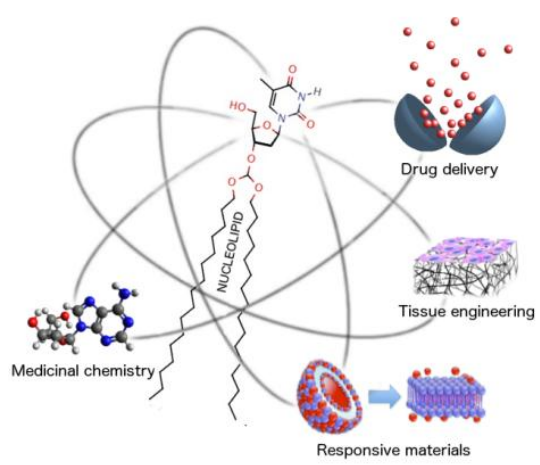

